\title{
Engineering the oleaginous yeast Yarrowia lipolytica to produce limonene from waste cooking oil
}

Yaru Pang, Yakun Zhao, Shenglong Li, Yu Zhao, Jian Li, Zhihui Hu, Cuiying Zhang, Dongguang Xiao

and Aiqun Yu* (1)

\begin{abstract}
Background: Limonene is an important biologically active natural product widely used in the food, cosmetic, nutraceutical and pharmaceutical industries. However, the low abundance of limonene in plants renders their isolation from plant sources non-economically viable. Therefore, engineering microbes into microbial factories for producing limonene is fast becoming an attractive alternative approach that can overcome the aforementioned bottleneck to meet the needs of industries and make limonene production more sustainable and environmentally friendly.
\end{abstract}

Results: In this proof-of-principle study, the oleaginous yeast Yarrowia lipolytica was successfully engineered to produce both $\mathrm{D}$-limonene and L-limonene by introducing the heterologous $\mathrm{D}$-limonene synthase from Citrus limon and L-limonene synthase from Mentha spicata, respectively. However, only $0.124 \mathrm{mg} / \mathrm{L}$ D-limonene and $0.126 \mathrm{mg} / \mathrm{L}$ L-limonene were produced. To improve the limonene production by the engineered yeast $Y$. lipolytica strain, ten genes involved in the mevalonate-dependent isoprenoid pathway were overexpressed individually to investigate their effects on limonene titer. Hydroxymethylglutaryl-CoA reductase (HMGR) was found to be the key rate-limiting enzyme in the mevalonate (MVA) pathway for the improving limonene synthesis in Y. lipolytica. Through the overexpression of HMGR gene, the titers of D-limonene and L-limonene were increased to $0.256 \mathrm{mg} / \mathrm{L}$ and $0.316 \mathrm{mg} / \mathrm{L}$, respectively. Subsequently, the fermentation conditions were optimized to maximize limonene production by the engineered $Y$. lipolytica strains from glucose, and the final titers of D-limonene and L-limonene were improved to $2.369 \mathrm{mg} / \mathrm{L}$ and $2.471 \mathrm{mg} / \mathrm{L}$, respectively. Furthermore, fed-batch fermentation of the engineered strains Po $1 \mathrm{~g} \mathrm{KdHR}$ and Po1g KIHR was used to enhance limonene production in shake flasks and the titers achieved for D-limonene and L-limonene were $11.705 \mathrm{mg} / \mathrm{L}(0.443 \mathrm{mg} / \mathrm{g})$ and $11.088 \mathrm{mg} / \mathrm{L}(0.385 \mathrm{mg} / \mathrm{g})$, respectively. Finally, the potential of using waste cooking oil as a carbon source for limonene biosynthesis from the engineered Y. lipolytica strains was investigated. We showed that D-limonene and L-limonene were successfully produced at the respective titers of $2.514 \mathrm{mg} / \mathrm{L}$ and $2.723 \mathrm{mg} / \mathrm{L}$ under the optimal cultivation condition, where $70 \%$ of waste cooking oil was added as the carbon source, representing a 20 -fold increase in limonene titer compared to that before strain and fermentation optimization.

Conclusions: This study represents the first report on the development of a new and efficient process to convert waste cooking oil into D-limonene and L-limonene by exploiting metabolically engineered Y. lipolytica strains for

\footnotetext{
${ }^{*}$ Correspondence: yuaiqun@tust.edu.cn

State Key Laboratory of Food Nutrition and Safety, Key Laboratory

of Industrial Fermentation Microbiology of the Ministry of Education,

Tianjin Key Laboratory of Industrial Microbiology, Tianjin Engineering

Research Center of Microbial Metabolism and Fermentation Process

Control, College of Biotechnology, Tianjin University of Science

and Technology, No.29 the 13th Street TEDA, Tianjin 300457, People's

Republic of China
}

(c) The Author(s) 2019. This article is distributed under the terms of the Creative Commons Attribution 4.0 International License (http://creativecommons.org/licenses/by/4.0/), which permits unrestricted use, distribution, and reproduction in any medium, provided you give appropriate credit to the original author(s) and the source, provide a link to the Creative Commons license, and indicate if changes were made. The Creative Commons Public Domain Dedication waiver (http://creativecommons.org/ publicdomain/zero/1.0/) applies to the data made available in this article, unless otherwise stated. 
fermentation. The results obtained in this study lay the foundation for more future applications of $Y$. lipolytica in converting waste cooking oil into various industrially valuable products.

Keywords: Yarrowia lipolytica, Limonene, Mevalonate pathway, Metabolic engineering, Fermentation optimization, Waste cooking oil

\section{Background}

Limonene, a monocyclic monoterpene, is an important biologically active natural product. It exists in two optical forms, i.e., D- and L-limonene with distinct properties and applications [1]. At present, limonene is widely used as a commercial flavor and fragrance in food, beverage, cosmetic and pharmaceutical industries because the $\mathrm{D}$ and $\mathrm{L}$ enantiomers possess pleasant lemon- or turpentine-like odor, respectively [2, 3]. Furthermore, $\mathrm{D}$-limonene is used as a natural, environmentally friendly abluent in machinery, printing, aviation and electronic devices $[4,5]$, while L-limonene is a precursor for the biosynthesis of L-menthol, which is the major component of mint $[6,7]$. In addition, limonene can also serve as a biofuel, a biopreservative, a versatile cleaning agent to replace toxic solvents and a key building block for a wide range of industrial products, including pharmaceuticals, nutraceuticals and other high-value fine chemicals [8-10]. Thus, there is an ever-increasing demand for limonene due to its vast range of applications. Currently, industrial production of limonene is mainly achieved by plant extraction, but the low abundance of limonene in plants renders their isolation from plant sources noneconomically viable [11]. Likewise, chemical syntheses of limonene are limited by the complexity of the production equipment and low conversion rate of raw materials. These processes are also energy intensive and can cause environmental issues. Therefore, alternative production routes are urgently needed for industrial production of limonene. A promising approach is the engineering of microbes into microbial cell factories for de novo biosynthesis of limonene, and a variety of microbes have been engineered to produce $\mathrm{D}$-limonene or $\mathrm{L}$-limonene in many previous researches (Table 1).

Yarrowia lipolytica, a non-conventional oleaginous yeast, is an attractive biochemical production host that can be employed for the production of food-grade products since it is classified by the US Food and Drug Administration as generally recognized as safe (GRAS) [12]. With the completion of the genome sequencing of $Y$. lipolytica and generation of available genetic tools for genetic manipulation, it has received increasing attention from researchers for use in metabolic engineering to produce valuable compounds. In recent years, $Y$. lipolytica has shown its versatility and importance as a production host by successfully demonstrating its utilization for a wide range for metabolic engineering and biotechnological applications [13-15].

In limonene-producing plants, limonene is biosynthesized from the precursor geranyl diphosphate (GPP) by enzymatic biotransformation with $d$ - or L-limonene synthetase (Fig. 1a) [16]. While plants produce GPP via the methylerythritol phosphate (MEP) pathway from pyruvate and glyceraldehyde-3-phosphate (Fig. 1a) [17, 18 ], yeasts rely on the mevalonate (MVA) pathway to produce GPP from acetyl-CoA (Fig. 1b). Considering these requirements for implementing the limonene biosynthesis pathway, Y. lipolytica is an excellent microbial production host because (1) being an oleaginous yeast with an abundant pool of intracellular acetyl-CoA [13], it has great potential to be rewired to overproduce GPP to serve as a precursor for biotransformation to limonene and (2) being an eukaryote, it is suited for heterologous expression of the plant-derived D- and L-limonene synthases. Moreover, a unique characteristic of Y. lipolytica is that it can grow on a wide range of cheap hydrophobic and hydrophilic carbon sources, including alkanes, alkenes, fats, alcohols and organic acids [12, 19-24], thus it can be used as an effective whole-cell biocatalyst to bioremediate polluted environments [25]. Notably, this organism has been exploited to utilize waste cooking oils (WCO) as carbon sources for growth and substrates for bioconversion $[26,27]$. Therefore, the biosynthesis of limonene from WCO with engineered Y. lipolytica can be a sustainable and economical avenue for production of the valuable and versatile chemical.

In this study, we demonstrated the engineering and optimization of $Y$. lipolytica toward limonene production from WCO. Y. lipolytica was first engineered to produce $\mathrm{D}$-limonene and $\mathrm{L}$-limonene by heterologous expression of selected D-limonene synthase gene and L-limonene synthase gene, respectively. Subsequently, ten genes involved in the MVA pathway were overexpressed to identify the key enzymes for improving the yields of D-limonene and L-limonene. To further enhance the yields of D-limonene and L-limonene, we varied the parameters for cultivating the engineered $Y$. lipolytica strains to optimize the fermentation conditions. Finally, the potential of using WCO as the carbon source for limonene production was investigated with the engineered Y. lipolytica strains. We envision that this work will provide insights in developing strategies for 


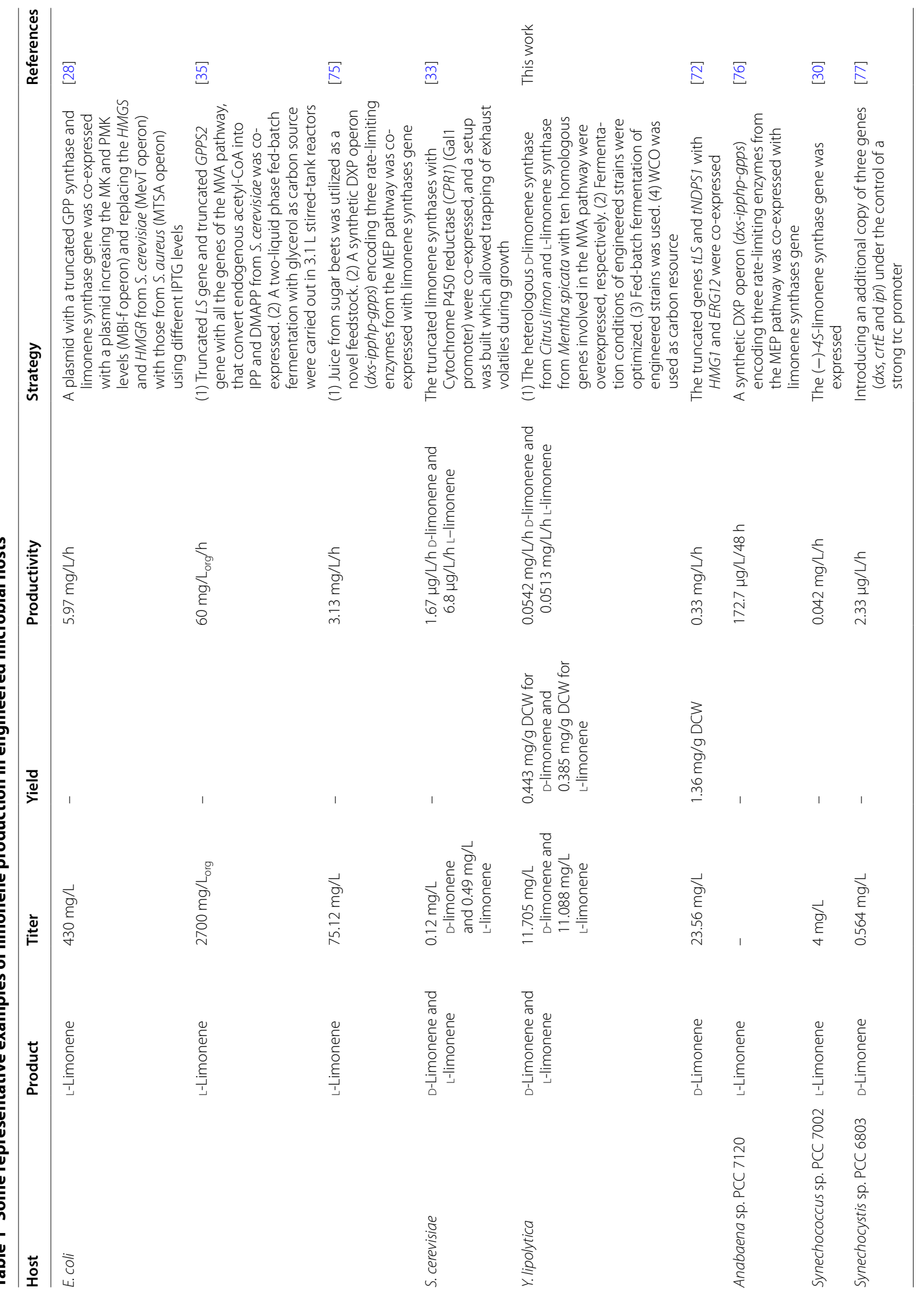



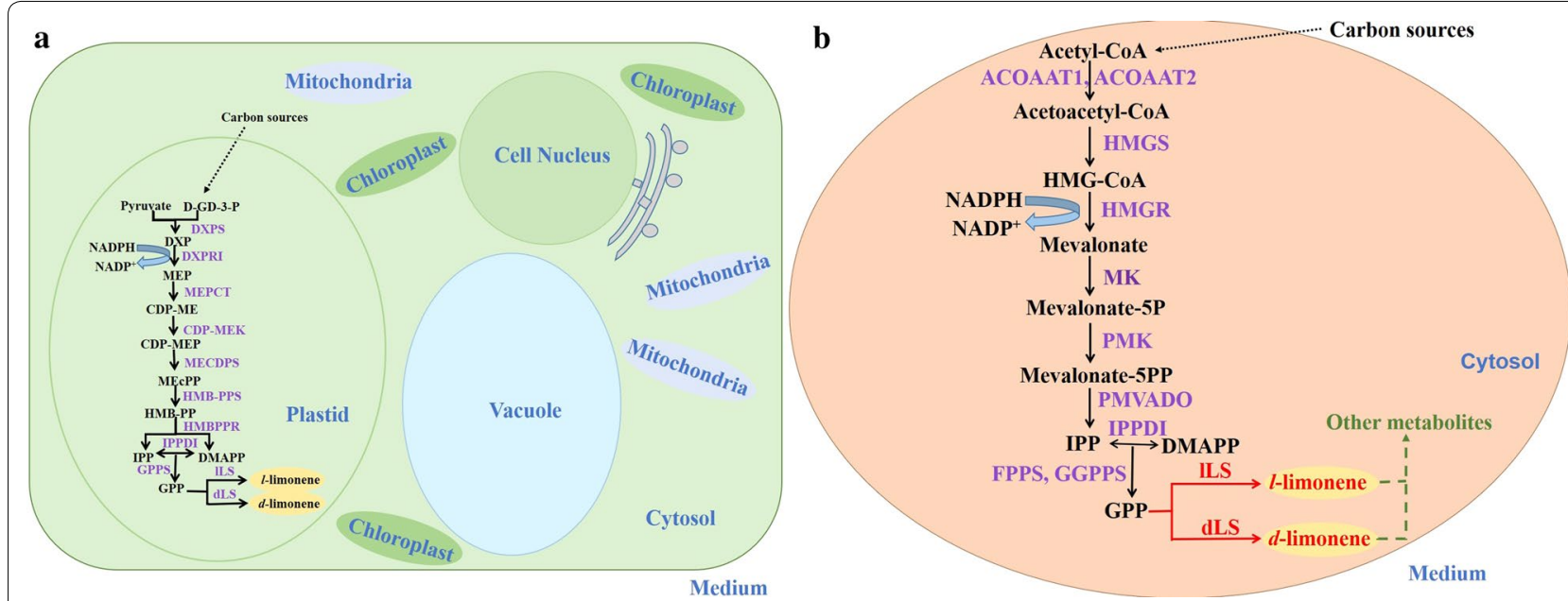

Fig. 1 Biosynthesis pathway for limonene production in plants and the yeast Y. lipolytica. Limonene is biosynthesized from the precursor GPP by enzymatic biotransformation with D- or L-limonene synthetase. IPP and DMAPP are converted to GPP in both plant and Y. lipolytica. a Biosynthesis pathway for limonene production in plants. Plants produce GPP via the methylerythritol phosphate pathway from pyruvate and glyceraldehyde-3-phosphate. b Biosynthesis pathway for limonene production in Y. lipolytica. Yeast rely on the mevalonate pathway to produce GPP from acetyl-COA. Since LS is not present in Y. lipolytica to construct a complete limonene pathway in Y. lipolytica, two heterologous genes encoding D-limonene synthase ( $d L S$, from C. limon) and L-limonene synthase gene (ILS, from M. spicata) were introduced (shown in red). And downstream metabolic pathways of limonene may lie in yeast (shown in blue). The endogenous MVA pathway enzymes (purple) that were overexpressed in the engineered Y. lipolytica strains. Enzymes involved in the MEP pathway in plants and in the MVA pathway in Y. lipolytica are shown in parentheses. Homologous enzymes found in Y. lipolytica are shown in purple. DXPS DXP synthase, DXPRI DXP-reductoisomerase, MEPCT MEP cytidylyltransferase, CDP-MEK CDP-ME kinase, MECDPS MECDP-synthase, HMBPPS (E)-4-hydroxy-3-methylbut-2-enyl-diphosphate synthase, HMBPPR HMBPP reductase, IPPDI isopentenyl-diphosphate delta-isomerase, GPPS geranyl-diphosphate synthase, ILS L-limonene synthase, $d L S \mathrm{D}$-limonene synthase, ACOAAT1 acetyl-CoA C-acetyltransferase 1, ACOAAT2 acetyl-CoA C-acetyltransferase 2, HMGS hydroxymethylglutaryl-CoA synthase, HMGR hydroxymethylglutaryl-CoA reductase, MK mevalonate kinase, PMK phosphomevalonate kinase, PMVADO diphosphomevalonate decarboxylase, IPPDI isopentenyl-diphosphate delta-isomerase, GGPPS geranylgeranyl diphosphate synthase, type III, FPPS farnesyl diphosphate synthase

exploiting Y. lipolytica as a microbial cell factory for sustainable and economical production of valuable chemicals from waste oleaginous feedstock.

\section{Results and discussion}

Heterologous production of limonene in Y. lipolytica through limonene synthase expression

Yarrowia lipolytica Po1g KU70s was used as the platform strain for limonene production in this study. This is because, in addition to having relatively high level of intracellular acetyl-CoA as aforementioned, the tolerance of this oleaginous strain to D-limonene or L-limonene was found to be much higher than that of the conventional yeast host Saccharomyces cerevisiae (Additional file 1: Fig. S1). Furthermore, it was observed that the rate of precise homologous recombination in the Polg KU70 $\Delta$ strain is much higher than that of the parent strain Po1g, which will facilitate genomic manipulation.

As Y. lipolytica is not a natural producer of limonene, heterologous enzymes need to be expressed to convert the GPP synthesized to limonene via the MVA pathway. To this end, D-limonene synthase gene $(d L S)$ from Citrus limon and L-limonene synthase gene (lLS) from Mentha spicata were selected for production of D-limonene and L-limonene, respectively, as $d L S$ gene and $l L S$ gene have been shown to be used successfully to produce D-limonene and L-limonene (Additional file 1: Fig. S2), respectively, in heterologous microbial hosts [28-32]. Subsequently, the codon-optimized $d L S$ gene and $l L S$ gene were individually integrated into the chromosome of Y. lipolytica Po1g KU70 $\Delta$ strain and expressed under the strong constructive promoter hp4d. The resulting engineered strains Po1g KdLS and Po1g KILS were cultured in the YPD medium. Overlaying with $n$-dodecane appears to be the most efficient method to recover the limonene production in $Y$. lipolytica, because this method has been proven very efficient for trapping large quantities of volatile substances accumulated in different microbes [30, 33-35], which may be exported by passive diffusion or active transporters [36, 37]. And it has no significant effects on the cell growth of $Y$. lipolytica (Additional file 1: Fig. S3). Thus, a 10\% $n$-dodecane overlay was first added to the culture to capture the volatile limonene. The 9-day time courses of D-limonene and L-limonene production titers by Po1g KdLS and Po1g KILS, respectively, are shown in Fig. 2. The titers of D-limonene and L-limonene increased continuously from the beginning of cultivation up to day 5 , reaching a 


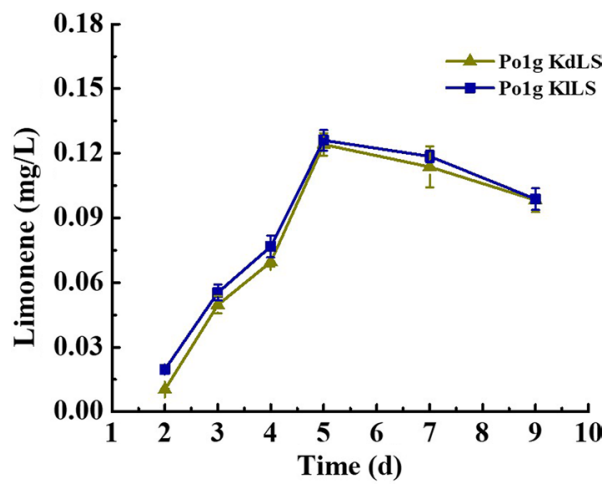

Fig. 2 Time course of D-limonene and L-limonene production in the engineered Y. lipolytica strains. The cells of Po1g KdLS and Po1g KILS were cultivated at $30^{\circ} \mathrm{C}$ and $200 \mathrm{rpm}$ with an initial $\mathrm{OD}_{600}$ of 0.1 in $30 \mathrm{~mL}$ of liquid YPD medium in a 250-mL shake flask, and limonene titers were determined at the 2-, 3-, 4-, 5-, 7- and 9-day time-points. To avoid loss of limonene during cultivation, $8 \%$ of $n$-dodecane overlay was added into the YPD medium prior to cultivation. _ _ : production of D-limonene by Po1g KdLS. ——: production of L-limonene by Po1g KILS. Bars represent limonene titers. All values presented are the mean of three biological replicates \pm standard deviation

peak titer of $0.124 \mathrm{mg} / \mathrm{L}$ for D-limonene and $0.126 \mathrm{mg} / \mathrm{L}$ for L-limonene. Subsequently, the titers of D-limonene and L-limonene declined gradually. This is probably because of ceasing production of limonene upon glucose depletion, as D-limonene and L-limonene titers further increased upon supplementation with additional glucose (Additional file 1: Fig. S4). It was previously reported that carbon starvation brings about an almost complete loss of fermentative capacity [38]. Subsequently, P450 monooxygenases present in $Y$. lipolytica for alkane assimilation may have oxidized the exocyclic methyl group of limonene and divert the flux to other side-products [39-41]. In addition, glucose depletion probably could reduce the transportation of limonene from inside of the cell to the outside by affecting multidrug resistance pump activity [42]. Hence, these results indicated the functional expression of the codon-optimized $d L S$ gene and $l L S$ gene in Y. lipolytica and these strains will serve as limonene-producing base strains for further engineering and optimization.

\section{Overexpression of endogenous genes involved in the MVA pathway of $Y$. lipolytica to further improve limonene production}

To further enhance limonene production following the successful biosynthesis of limonene in Y. lipolytica, the genes involved in the MVA pathway were overexpressed in an attempt to increase the flux towards limonene. Ten genes, consisting of ACOAAT1, ACOAAT2, HMGS,
HMGR, MK, PMK, PMVADO, IPPDI, GGPPS and FPPS (Fig. 1b), were overexpressed individually and investigated for their effects on limonene overproduction to determine the key genes that are critical for limonene biosynthesis in the MVA pathway. To this end, twenty strains overexpressing the codon-optimized $d L S$ or $l L S$ gene with one of the ten endogenous genes in the MVA pathway of $Y$. lipolytica were constructed; all the genes were integrated into the chromosome of $Y$. lipolytica Po1g KU70 $\Delta$ and expressed with the strong constitutive promoter hp4d. The transcription levels of the genes were determined by qRT-PCR and verified to have increased, suggesting that all the ten individually integrated genes were overexpressed (Fig. 3). The twenty engineered strains were cultured in YPD medium for 5 days in shake flasks. Individual overexpression of the selected genes did not cause any adverse effect on cell growth (Additional file 1: Fig. S5). The limonene titers of the strains showed that the overexpression of the individual corresponding genes could improve the limonene production compared to control strains expressing only the respective limonene synthases (Fig. 3). The HMGR-overexpressed strains, Polg KdHR (Fig. 3a) and Po1g KlHR (Fig. 3b), achieved the highest titers of $46.703 \mu \mathrm{g} / \mathrm{g} \mathrm{DCW}(0.286 \mathrm{mg} / \mathrm{L})$ and $63.418 \mu \mathrm{g} / \mathrm{g}$ DCW $(0.367 \mathrm{mg} / \mathrm{L})$ for $\mathrm{D}$-limonene and L-limonene after 5 days of cultivation, respectively. These represent a $288 \%$ increase in D-limonene titer over the Po1g KdLS strain and a 299\% increase in L-limonene titer over the Po1g KILS strain. However, the titer change in limonene was not consistent with the change in the levels of gene transcription, suggesting that the highest limonene titer, which was achieved by the overexpression of HMGR, was not attributed to the highest increase in gene expression level (Fig. 3). Our results indicate that the 3-hydroxy-3-methylglutaryl-coenzyme A (HMGCoA) reductase of $Y$. lipolytica encoded by HMGR is the key regulatory step controlling isoprenoid metabolism, which improved limonene synthesis in Y. lipolytica upon overexpression. In numerous studies, HMG-CoA reductase has been proven to be a rate-limiting step in the production of the wide variety of molecules derived from mevalonate by the mevalonate pathway [43-47]. Thus, the strains Po1g KdHR and Po1g KlHR were chosen for further enhancement in limonene production by fermentation optimization.

\section{Fermentation optimization of the engineered $Y$. lipolytica strains}

In microorganisms, the biosynthesis of most metabolites is strongly affected by fermentation parameters such as temperature, $\mathrm{pH}$, rotation speed, initial cell density, additives and nutrients. We, therefore, hypothesized that the capability of our engineered $Y$. lipolytica strains in 

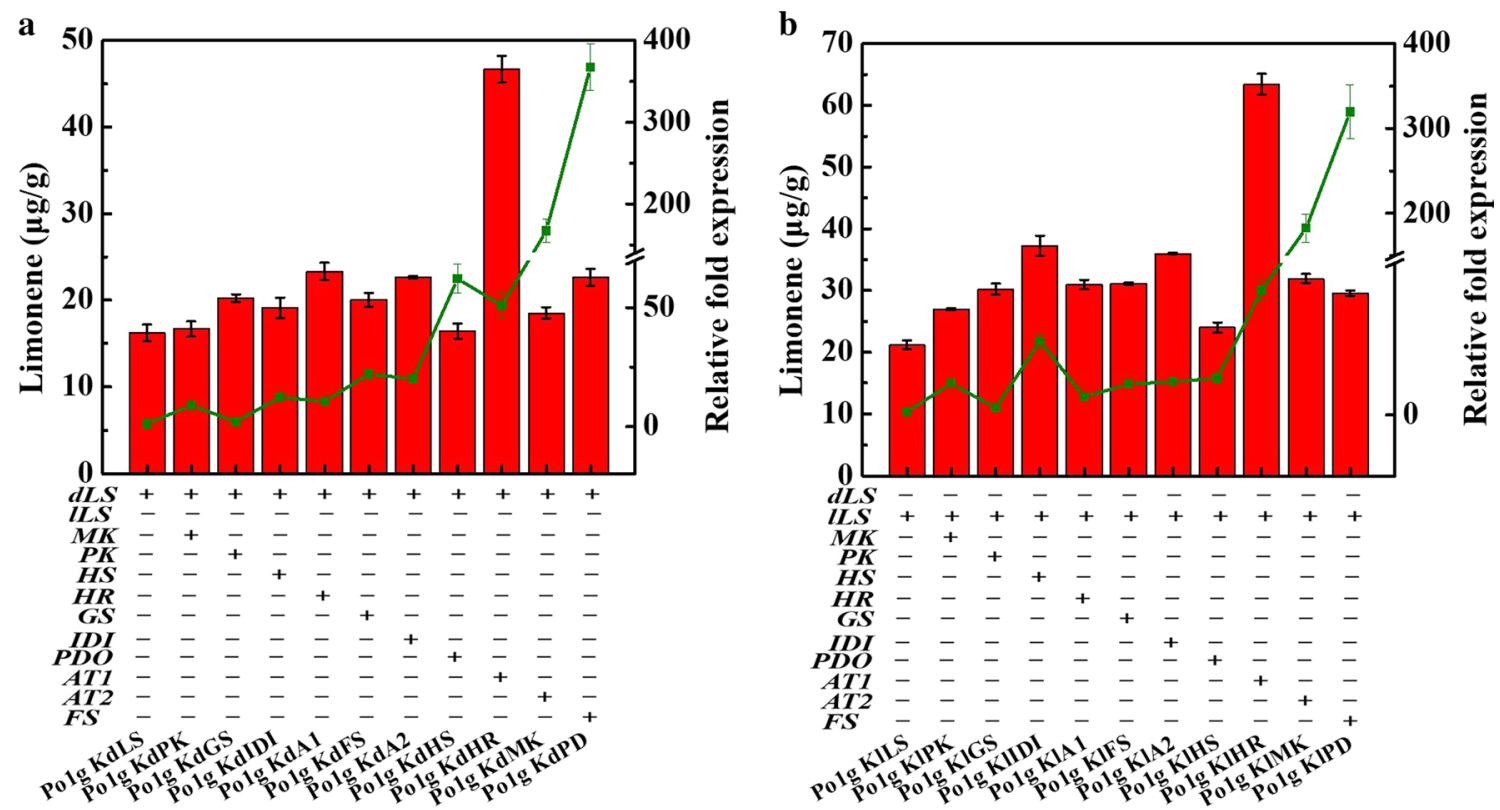

Fig. 3 Effects of single-gene overexpression of genes involved in the MVA pathway on D-limonene or L-limonene production. a Effects of single-gene overexpression of genes involved in the MVA pathway on D-limonene production. Ten genes including ACOAAT1, ACOAAT2, HMGS, HMGR, MK, PMK, PMVADO, IPPDI, GGPPS and FPPS involved in MVA pathway were overexpressed individually with D-limonene synthase. $\mathbf{b}$ Effects of single-gene overexpression of genes involved in the MVA pathway on L-limonene production. Ten genes including ACOAAT1, ACOAAT2, HMGS, HMGR, MK, PMK, PMVADO, IPPDI, GGPPS and FPPS involved in MVA pathway were overexpressed individually with L-limonene synthase. Bars represent limonene yields and lines represent gene expression improvements over controls. Titers of D-limonene or L-limonene were quantified after 5 days of cultivation and the RNAs of ten genes were extracted after 3 days of cultivation in shake flasks with $30 \mathrm{~mL}$ of liquid YPD medium. Glucose was used as the carbon source. The $d L S$ - or ILS-integrated strain cultivated in parallel was used as controls, and the gene encoding the $\beta$-actin protein was used as an internal standard. Relative gene expression measured relative to $\beta$-actin and normalized to controls was calculated as $2^{-\Delta \Delta C T}$. All values presented are the mean of three biological replicates \pm standard deviation

producing limonene could be further improved by optimizing the fermentation conditions. To our knowledge, this is the first report on the optimization of fermentation conditions for limonene production with microbes.

\section{Effect of temperature on the accumulation of limonene produced by the engineered $Y$. lipolytica strains}

Temperature, one of the important environmental factors for gene expression and enzyme activity that are associated with microbial cell growth and metabolism, is often one of the key parameters affecting microbial fermentation performance $[48,49]$. To investigate the effect of cultivation temperature on the accumulation of D-limonene and L-limonene during glucose fermentation by the engineered Po1g KdHR and Po1g KlHR strains, the cells were cultivated at $200 \mathrm{rpm}$ with an initial $\mathrm{OD}_{600}$ of 0.1 and $8 \%$ of $n$-dodecane in $30 \mathrm{~mL}$ of liquid YPD medium in a 250-mL shake flask for 5 days at various cultivation temperatures, i.e., $15,20,25$, and $30{ }^{\circ} \mathrm{C}$. As shown in Fig. 4a, while there was a slight decrease in the biomass of Polg KdHR and Po1g KlHR when the cultivation temperature was increased from 15 to $20^{\circ} \mathrm{C}$, the titers of D-limonene and L-limonene increased sharply. However, increasing the cultivation temperature from 20 to $30{ }^{\circ} \mathrm{C}$ drastically reduced the titers for both strains. Therefore, after 5 days of cultivation, the maximum limonene titers $(0.427 \mathrm{mg} / \mathrm{L}$ for D-limonene and $0.632 \mathrm{mg} / \mathrm{L}$ for L-limonene) were achieved from the Po1g KdHR and Po1g KlHR strains, respectively, at $20{ }^{\circ} \mathrm{C}$, suggesting that this is the optimum temperature for the production of $\mathrm{D}$-limonene and L-limonene from the engineered Y. lipolytica strains. It is well known that low temperature is beneficial for decreasing the evaporation of volatile substances, and it was also demonstrated that the evaporation of limonene was decreased at low temperature (Additional file 1: Fig. S6). Therefore, it is $20^{\circ} \mathrm{C}$ not $15{ }^{\circ} \mathrm{C}$ nor $30{ }^{\circ} \mathrm{C}$ that improved limonene production, which can probably be attributed to the combined effect of increased fermentation yield of limonene and the suppression of evaporation.

\section{Effect of rotation speed on the accumulation of limonene produced by the engineered $Y$. lipolytica strains}

Rotation speed plays a very important role in aerobic fermentation because it influences the mass transfer of gases, such as $\mathrm{O}_{2}$ and $\mathrm{CO}_{2}$, in and out of the growth medium [50]. Therefore, the effect of rotation speed 

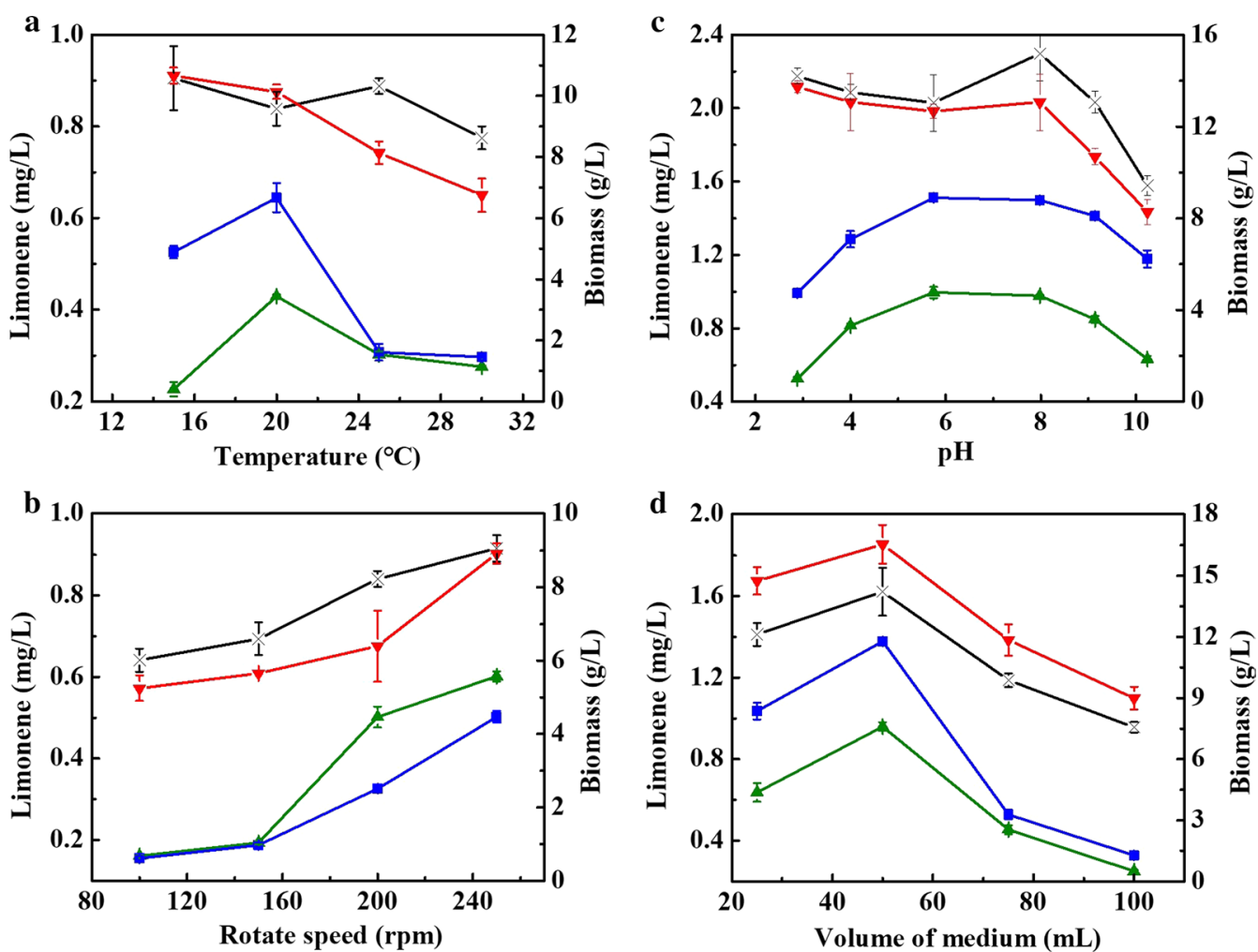

$\longrightarrow$ Po1g KdHR Con $\longrightarrow$ Po1g KIHR Con $\longrightarrow$ Po1g KdHR Bio $\longrightarrow$ Po1g KIHR Bio

Fig. 4 Effect of temperature, initial pH, rotation speed and volume of medium on D-limonene or L-limonene accumulation of Po1g KdHR or Po $1 \mathrm{~g}$ KIHR. a Effect of temperature on D-limonene or L-limonene accumulation of Po1g KdHR or Po1g KIHR. The cultivation was performed at $200 \mathrm{rpm}$ with an initial $\mathrm{OD}_{600}$ of 0.1 and $8 \%$ of $n$-dodecane in $30 \mathrm{~mL}$ of liquid YPD medium in a 250-mL shake flask for 5 days at different temperatures (15, 20,25 and $\left.30^{\circ} \mathrm{C}\right) \cdot \mathbf{b}$ Effect of rotation speed on D-limonene or L-limonene accumulation of Po1 $\mathrm{g} \mathrm{KdHR}$ or Po1g KIHR. The cells were cultivated at $30^{\circ} \mathrm{C}$ with an initial $\mathrm{OD}_{600}$ of 0.1 and $8 \%$ of $n$-dodecane in $30 \mathrm{~mL}$ of liquid YPD medium in a 250-mL shake flask for 5 days at different rotation speeds (100 rpm, 150 rpm, 200 rpm and 250 rpm). c Effect of initial pH on D-limonene or L-limonene accumulation of Po1g KdHR or Po1g KIHR. The cultivation was performed at the optimum fermentation temperature of $20^{\circ} \mathrm{C}$ and the optimum rotation speed of $250 \mathrm{rpm}$, with an initial $\mathrm{OD}_{600}$ of 0.1 and $8 \%$ of $n$-dodecane in $30 \mathrm{~mL}$ of liquid YPD medium in a $250-\mathrm{mL}$ shake flask for 5 days at six different initial pH values ranging from 3 to 10 . $\mathbf{d}$ Effect of volume of medium on D-limonene or L-limonene accumulation of Po1g KdHR or Po1g KIHR. The cultivation was performed at the optimum fermentation temperature of $20^{\circ} \mathrm{C}$, the optimum rotation speed of $250 \mathrm{rpm}$, with an initial $\mathrm{OD}_{600}$ of 0.1 and $8 \%$ of $n$-dodecane in a 250 - $\mathrm{mL}$ shake flask for 5 days at different volumes of liquid YPD medium $(25 \mathrm{~mL}, 50 \mathrm{~mL}, 75 \mathrm{~mL}$ and $100 \mathrm{~mL})$. ___ D-limonene accumulation of Po1g KdHR; $\longrightarrow$ - L-limonene accumulation of Po1g KIHR; ——: biomass accumulation of Po1g KdHR; ——: biomass accumulation of Po1g KIHR. All values presented are the mean of three biological replicates \pm standard deviation

on the accumulation of D-limonene and L-limonene by the engineered Po1g KdHR and Po1g KlHR strains was herein investigated. To this end, the cultivation was performed at $30{ }^{\circ} \mathrm{C}$ with an initial $\mathrm{OD}_{600}$ of 0.1 and $8 \%$ of $n$-dodecane in $30 \mathrm{~mL}$ of liquid YPD medium in a $250-\mathrm{mL}$ shake flask for 5 days while four different rotation speeds $(100,150,200$, and $250 \mathrm{rpm})$ were applied to the cultures. As shown in Fig. 4b, the biomass gradually increased and limonene production progressively improved as the rotation speed increased from 100 to $250 \mathrm{rpm}$, at which the maximum titers of limonene $(0.603 \mathrm{mg} / \mathrm{L}$ for $\mathrm{D}$-limonene and $0.509 \mathrm{mg} / \mathrm{L}$ for L-limonene) were achieved from Polg KdHR and
Po1g KlHR. Therefore, these results imply that the improved titers of limonene may be attributed to the increased biomass. However, the effect of rotation speed over $250 \mathrm{rpm}$ was not tested because of the speed limit of the routine incubator shaker used in this study. Thus, the optimum rotation speed for production of D-limonene and L-limonene from the engineered $Y$. lipolytica strains was established to be $250 \mathrm{rpm}$. These results suggest that high rotation speed in shake flask cultures is imperative to ensure sufficient oxygen supply for the optimum production of limonene by the strictly aerobic yeast $Y$. lipolytica [51]. 


\section{Effect of $\mathrm{pH}$ on the accumulation of limonene produced by the engineered $Y$. lipolytica strains}

Extracellular $\mathrm{pH}$ is often a critical parameter to obtain optimal microbial activity in the culture media. In particular, $\mathrm{pH}$ changes directly influence membrane potential, proton motive force and consequently substrate utilization and product profile [49]. In this regard, the $\mathrm{pH}$ change in the medium during time of the culture was measured. We found that the $\mathrm{pH}$ of the fermentation medium did not significantly change at the initial stage of the Y. lipolytica cultivation (from day 1 to day 3, Additional file 1: Fig. S7), although the $\mathrm{pH}$ rose to around 8.5 at the end of the cultivation. Therefore, we propose initial $\mathrm{pH}$ could be a limiting factor for limonene production in the process of fermentation by engineered $Y$. lipolytica strains. To verify this hypothesis, six different initial $\mathrm{pH}$ values ranging from 3 to 10 were used to determine the effect of initial $\mathrm{pH}$ value of the YPD medium on the accumulation of $\mathrm{D}$-limonene and L-limonene by the engineered Y. lipolytica Po1g KdHR and Po1g KlHR strains. The cultivation was performed at the optimum fermentation temperature of $20{ }^{\circ} \mathrm{C}$ and the optimum rotation speed of $250 \mathrm{rpm}$, with an initial $\mathrm{OD}_{600}$ of 0.1 and $8 \%$ of $n$-dodecane in $30 \mathrm{~mL}$ of liquid YPD medium in a $250-\mathrm{mL}$ shake flask for 5 days. Limonene production by the engineered $Y$. lipolytica strains was possible over a wide range of $\mathrm{pH}$ values but the titers varied. As shown in Fig. 4c, when the $\mathrm{pH}$ was raised from 2.88 to 5.74 , there was a slight decrease in biomass as the titers of D-limonene and L-limonene increased gradually to peak at pH 5.74. Raising the $\mathrm{pH}$ from 5.74 and 7.98 increased the biomass but the titers of both D-limonene and L-limonene were almost constant. Further increasing the $\mathrm{pH}$ from 7.98 to 10.24 reduced both the biomass and the titers. In general, the reason why the highest titers of both D-limonene and $\mathrm{L}$-limonene were obtained at $\mathrm{pH} 5.74-7.98$ is that these $\mathrm{pHs}$ are close to the physiological $\mathrm{pH}$ and a large deviation of the extracellular environment from the physiological $\mathrm{pH}$ can lead to changes in cellular gene expression, enzyme activity, signaling pathways and ultimately the biological activity of microorganisms. Since the highest limonene titers were achieved at $\mathrm{pH} 5.74$, this $\mathrm{pH}$ was established to be optimum $\mathrm{pH}$ for production of D-limonene and L-limonene from the engineered Y. lipolytica strains.

\section{Effect of the volume of culture medium on the accumulation of limonene produced by the engineered $Y$. lipolytica strains}

As mentioned above, the oleaginous yeast $Y$. lipolytica is a strictly aerobic yeast. During the cultivation process, different levels of dissolved oxygen in the medium can greatly influence cell growth of this yeast, and thus production of target products. Similar to rotation speed, the volume of liquid culture medium is another factor that can affect the level of dissolved oxygen in the medium. In general, the level of dissolved oxygen in the medium would be high when the volume of culture medium is low. However, low culture volume could also lead to insufficient supply of nutrient and energy. To study the effect of the volume of liquid YPD medium on the accumulation of D-limonene and L-limonene, the engineered $Y$. lipolytica strains were cultivated in different volumes of liquid YPD medium $(25 \mathrm{~mL}, 50 \mathrm{~mL}, 75 \mathrm{~mL}$, and $100 \mathrm{~mL}$ ) at the optimum fermentation temperature of $20^{\circ} \mathrm{C}$, the optimum rotation speed of $250 \mathrm{rpm}$, with an initial $\mathrm{OD}_{600}$ of 0.1 and $8 \%$ of $n$-dodecane in a $250-\mathrm{mL}$ shake flask for 5 days. As shown in Fig. 4d, the biomass and the titers of both D-limonene and L-limonene were enhanced sharply when the culture volume was increased from 25 to $50 \mathrm{~mL}$ but further increase in culture volume to 75 and $100 \mathrm{~mL}$ caused drastic decrease in the biomass and titers. The maximum titers of limonene $(1.045 \mathrm{mg} / \mathrm{L}$ for D-limonene and $1.530 \mathrm{mg} / \mathrm{L}$ for L-limonene) were achieved at the peak of biomass from $50 \mathrm{~mL}$ of YPD medium, hence implying that the improved titers can be attributed to the increased biomass. These results also indicate that $50 \mathrm{~mL}$ of YPD medium is the optimum culture volume for both availability of dissolved oxygen and supply of nutrients in 250-mL shake flasks for the production of D-limonene and L-limonene from the engineered $Y$. lipolytica strains. To further investigate the effects of different levels of dissolved oxygen in the medium on the accumulation of D-limonene and L-limonene during glucose fermentation by the engineered Po1g KdHR and Po1g KlHR strains, the fermentation was carried out in a 5-L laboratory fermenter. However, the dissolved oxygen level was unstable during the batch fermentation of the engineered $Y$. lipolytica strains (Additional file 1: Fig. S8). One possible reason is that the oxygen consumption by the yeast cells was too fast for the effects of culture volume and rotational speed to be stable. In addition, it is possible that inappropriate types and volumes of antifoaming agents added caused the phenomenon. The effect of different dissolved oxygen level on the accumulation of limonene was thus not determined by this experiment in fermenter.

\section{Effect of the volume of $n$-dodecane on the accumulation of limonene produced by the engineered Y. lipolytica strains} Limonene, a highly volatile monoterpene, can be relatively easy to be captured by overlaying the microbial fermentation system with $n$-dodecane $[30,34,35]$. Although $n$-dodecane is a known carbon source for Y. lipolytica, it did not contribute significantly to the growth of our engineered strains in the rich medium that we used for cultivation (Additional file 1: Fig. S3). However, the optimum volume of $n$-dodecane overlay for efficient capturing of 
Fig. 5 Effect of volume of $n$-dodecane, initial $\mathrm{OD}_{600}$ and $\mathrm{MgSO}_{4} \cdot 7 \mathrm{H}_{2} \mathrm{O}$ on D-limonene or L-limonene accumulation in Polg KdHR or Polg KIHR. a Effect of volume of $n$-dodecane on D-limonene or L-limonene accumulation in Po1g KdHR or Po1g KIHR. The cells were cultivated at the optimum fermentation temperature of $20^{\circ} \mathrm{C}$, the optimum rotation speed of $250 \mathrm{rpm}$, with an initial $\mathrm{OD}_{600}$ of 0.1 in $30 \mathrm{~mL}$ of liquid YPD medium in a 250-mL shake flask for 5 days with different volumes of $n$-dodecane $(6 \%, 8 \%, 10 \%$, and $12 \%)$. b Effect of initial $\mathrm{OD}_{600}$ on D-limonene or L-limonene accumulation in Po1g KdHR or Polg KIHR. The cells were cultivated at the optimum fermentation temperature of $20^{\circ} \mathrm{C}$, the optimum rotation speed of $250 \mathrm{rpm}$, with an initial $\mathrm{OD}_{600}$ of 0.1 and $8 \%$ of $n$-dodecane in $30 \mathrm{~mL}$ of liquid YPD medium in a 250-mL shake flask for 5 days with different initial $\mathrm{OD}_{600}\left(0.01,0.1,1.0\right.$ and 2.0). c Effect of volume of $\mathrm{MgSO}_{4} \cdot 7 \mathrm{H}_{2} \mathrm{O}$ on D-limonene or L-limonene accumulation in Po1g KdHR or Po1g KIHR. The cultivation was performed at the optimum fermentation temperature of $20^{\circ} \mathrm{C}$, the optimum rotation speed of $250 \mathrm{rpm}$, the optimum initial $\mathrm{OD}_{600}$ of 2.0 , the optimum $\mathrm{pH}$ of 5.74 and the best $n$-dodecane volume $10 \%$ in the optimal $50 \mathrm{~mL}$-final-volume of medium in a 250-mL shake flask for 5 days with different concentrations of $\mathrm{MgSO}_{4} \cdot 7 \mathrm{H}_{2} \mathrm{O}(0,0.1 \%, 0.2 \%$, and $0.3 \%)$. _ _ : D-limonene accumulation of Polg KdHR; ——: L-limonene accumulation of Polg KIHR; ——: biomass accumulation of Polg KdHR; $\longrightarrow$ : biomass accumulation of Po1g KIHR. All values presented are the mean of three biological replicates \pm standard deviation

the limonene products from the fermentation broth of the engineered $Y$. lipolytica has not been determined. In this study, we investigated the efficiency of different $n$-dodecane overlay volume $(6 \%, 8 \%, 10 \%$ and $12 \%)$ in capturing the limonene from the fermentation broth of Po1g KdHR and Po1g KlHR. The cells were cultivated at the optimum fermentation temperature of $20{ }^{\circ} \mathrm{C}$ and the optimum rotation speed of $250 \mathrm{rpm}$, with an initial $\mathrm{OD}_{600}$ of $0.1 \mathrm{in} 30 \mathrm{~mL}$ of liquid YPD medium in a $250-\mathrm{mL}$ shake flask for 5 days. As shown in Fig. 5a, increasing the volume of $n$-dodecane from 6 to $10 \%$ only led to a marginal improvement in the titers of $\mathrm{D}$-limonene and L-limonene; further increase in $n$-dodecane volume to $12 \%$ decreased the limonene titer slightly. Hence, these results demonstrated that an excess of $n$-dodecane could not improve the capturing efficiency of the produced limonene. Since the highest titers of $\mathrm{D}$-limonene and L-limonene were achieved with $10 \% n$-dodecane, this was chosen as the optimum overlay volume for capturing the limonene produced from the engineered Y. lipolytica strains.

\section{Effect of initial $O D_{600}$ on the accumulation of limonene produced by the engineered $Y$. lipolytica strains}

In many previous reports, using a relatively high initial $\mathrm{OD}_{600}$ is a successful strategy to reduce the fermentation time and overcome the inhibition by inhibitors present in the microbial fermentation system. However, the higher initial $\mathrm{OD}_{600}$ could result in the problems such as decreased dissolved oxygen level and increased
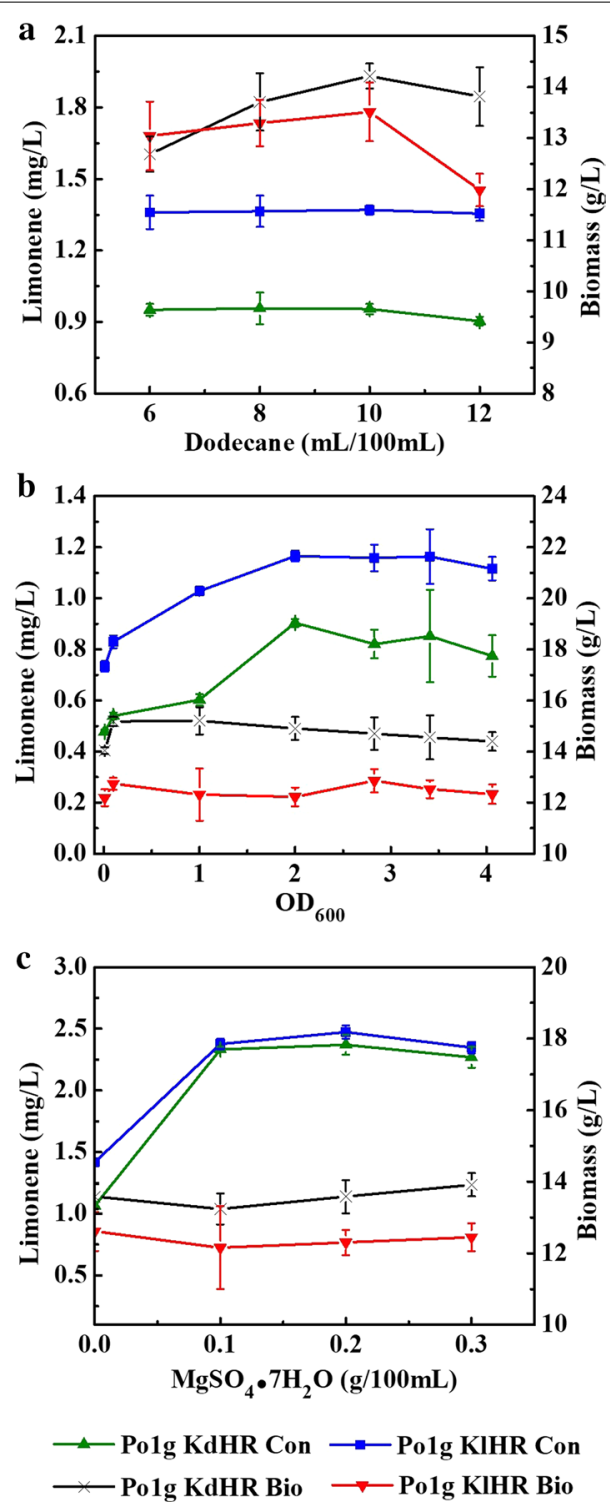

by-product level in the microbial fermentation system $[49,52-55]$. Therefore, the effect of initial $\mathrm{OD}_{600}$ on the accumulation of $\mathrm{D}$-limonene and L-limonene by the engineered Y. lipolytica strains was herein investigated. The Polg KdHR and Po1g KlHR strains were inoculated at different initial $\operatorname{OD}_{600}(0.01,0.1,1.0$, and 2.0) and cultivated at the optimum fermentation temperature of $20{ }^{\circ} \mathrm{C}$ and the optimum rotation speed of $250 \mathrm{rpm}$, with $8 \%$ of $n$-dodecane in $30 \mathrm{~mL}$ of liquid YPD medium in a $250-\mathrm{mL}$ shake flask for 5 days. As shown in Fig. 5b, the titers of both D-limonene and L-limonene were improved significantly when the initial $\mathrm{OD}_{600}$ was increased from 0.01 to 2.0 , although the increase was more drastic in the range of $0.01-0.1$ and more modest from 0.1 to 2.0. The titers 
of both D-limonene and L-limonene were not varied significantly when the initial $\mathrm{OD}_{600}$ was increased further from 2.0 to 4.06. In contrast, the biomass in general was not significantly varied. However, the fermentation time was not reduced and the biomass was not significantly varied with the increase of initial cell concentration. The maximum titer of limonene $(0.704 \mathrm{mg} / \mathrm{L}$ for $\mathrm{D}$-limonene and $1.265 \mathrm{mg} / \mathrm{L}$ for L-limonene) was obtained from Po1g KdHR and Po1g KlHR when the initial $\mathrm{OD}_{600}$ was 2.0, suggesting that this was the optimum initial $\mathrm{OD}_{600}$ for the production of $\mathrm{D}$-limonene and L-limonene from the engineered Y. lipolytica strains.

\section{Effect of $\mathrm{Mg}^{2+}$ concentration on the accumulation of limonene produced by the engineered Y. lipolytica strains}

Essential trace elements and metallic salts are necessary for growth and metabolism of microorganisms, thus have effects on the intracellular accumulation of metabolites in microorganisms $[56,57]$. Previously, it was proven that the addition of $\mathrm{Mg}^{2+}$ has great influence on fermentation performance of oleaginous microorganisms [58]. We thus hypothesized that the addition of $\mathrm{Mg}^{2+}$ will further enhance limonene production by the engineered $Y$. lipolytica strains. To verify this hypothesis, the culture medium was supplemented with different concentrations of $\mathrm{MgSO}_{4} \cdot 7 \mathrm{H}_{2} \mathrm{O}(0 \%, 0.1 \%, 0.2 \%$, and $0.3 \%)$. First, the effect of $\mathrm{Mg}^{2+}$ on growth of $Y$. lipolytica was investigated, and the results suggested that the effect of exogenous magnesium on the growth of the engineered $Y$. lipolytica strains was not significant under our experimental conditions (Additional file 1: Fig. S9). Then, Po1g KdHR and Po1g KlHR were cultivated in 250-mL shake flasks for 5 days with the optimum fermentation parameters determined afore, i.e., $50 \mathrm{~mL}$ of liquid YPD medium, temperature of $20{ }^{\circ} \mathrm{C}$, rotation speed of $250 \mathrm{rpm}$, initial $\mathrm{OD}_{600}$ of 2.0 , initial $\mathrm{pH}$ of 5.74 and $10 \% n$-dodecane overlay. The results showed that the biomass was not significantly varied and the titers of D-limonene and L-limonene in the medium supplemented with $\mathrm{Mg}^{2+}$ were much higher than those without added $\mathrm{Mg}^{2+}$ (Fig. 5c). The maximum titers of limonene $(2.268 \mathrm{mg} / \mathrm{L}$ for D-limonene and $2.347 \mathrm{mg} / \mathrm{L}$ for L-limonene) were achieved by Polg KdHR and Polg KlHR, respectively, with a supplement of additional $0.2 \%$ of $\mathrm{MgSO}_{4} \cdot 7 \mathrm{H}_{2} \mathrm{O}$, indicating that this is the optimum $\mathrm{Mg}^{2+}$ concentration for the production of D-limonene and L-limonene from the engineered $Y$. lipolytica strains. These results obtained could possibly be due to the fact that $\mathrm{Mg}^{2+}$ could enhance the activities of enzymes in the MVA pathway or limonene synthases in the engineered Y. lipolytica strains, which has been reported in the literature $[29,59] . \mathrm{Mn}^{2+}$ was also considered as a factor that may affect the activity of enzymes in the MVA pathway or limonene synthases as described

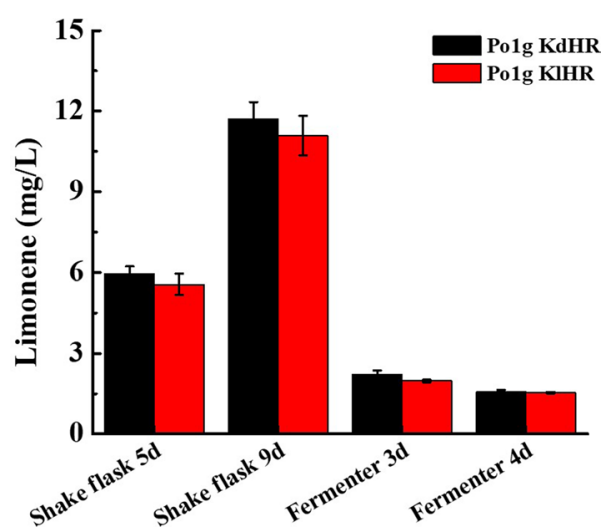

Fig. 6 D-Limonene or L-limonene accumulation in Polg KdHR or Po1g KIHR during fed-batch cultures on glucose. Fed-batch shake flask cultivation was carried out at $20^{\circ} \mathrm{C}, 250 \mathrm{rpm}$. The fermentation condition of fed-batch fermentation in the fermenter was performed at $28{ }^{\circ} \mathrm{C}, \mathrm{pH} 5.7$ and with $20 \%$ dissolved oxygen. $50 \%$ glucose solution was continuously fed into the shake flask and fermenter $(1 \mathrm{~mL}$ at every 24-h time-point and $120 \mathrm{~mL}$ over every 24 -h cultivation period, respectively). All values presented are the mean of three biological replicates \pm standard deviation

in some previous reports $[31,60]$. Therefore, the effect of $\mathrm{Mn}^{2+}$ on the accumulation of limonene produced by the engineered $Y$. lipolytica strains was also investigated. The results suggest that the addition of $\mathrm{Mn}^{2+}$ has no significant effect on the enhancement of limonene production and even decreased limonene titer at high concentrations of $\mathrm{Mn}^{2+}$ (Additional file 1: Fig. S10). These results suggest that it is $\mathrm{Mg}^{2+}$ and not $\mathrm{Mn}^{2+}$ that improved the production of D-limonene and L-limonene more in this work. In addition, the carbon/nitrogen ratio was investigated by changing the concentration of peptone, the results suggested that the optimum peptone concentration was $2 \%$ $(20 \mathrm{~g} / \mathrm{L})$, and this optimum peptone concentration was the concentration that we have used for the all the experiments (Additional file 1: Fig. S11).

Subsequently, fed-batch fermentation of the engineered strains Po1g KdHR and Po1g KlHR was used to enhance limonene production in shake flasks and a $5 \mathrm{~L}$-fermenter. After 9 days of cultivation in fed-batch fermentation of the engineered strains Po1g KdHR and Po1g KlHR in shake flasks, the limonene titers were determined and adjusted to the initial culture volume to account for the loss of growth medium and $n$-dodecane from evaporation; the remaining volume of culture was $43 \mathrm{~mL}$, the remaining volume of $n$-dodecane was $4.2 \mathrm{~mL}$, and $\mathrm{pH}$ of the cell culture was 7.8. As shown in Fig. 6, the titers of both D-limonene and L-limonene improved significantly upon fed-batch fermentation of the engineered strains Polg KdHR and Po1g KlHR. The titers of D-limonene and L-limonene further increased when the fermentation time was lengthened to 9 days in shake flasks, leading 
to D-limonene and L-limonene titers of $11.705 \mathrm{mg} / \mathrm{L}$ $(0.443 \mathrm{mg} / \mathrm{g})$ and $11.088 \mathrm{mg} / \mathrm{L}(0.385 \mathrm{mg} / \mathrm{g})$, respectively (the productivities of $\mathrm{D}$-limonene and $\mathrm{L}$-limonene were $0.0542 \mathrm{mg} / \mathrm{L} / \mathrm{h}$ and $0.0513 \mathrm{mg} / \mathrm{L} / \mathrm{h}$, respectively). These enhanced titers represent over 3.5-fold improvement compared with the titers of $2.369 \mathrm{mg} / \mathrm{L} \mathrm{D}$-limonene and $2.471 \mathrm{mg} / \mathrm{L} \mathrm{L}$-limonene before using fed-batch fermentation. Fed-batch fermentation at a constant glucose feed rate was carried out in a 5 - $\mathrm{L}$ fermenter, as it is an efficient measure to control oxygen consumption and ensure favorable cell growth [61]. The data of limonene fedbatch fermentation parameters in the 5-L fermenter are shown in Additional file 1: Fig. S12. However, the titers of both D-limonene and L-limonene declined markedly when fed-batch fermentation of the engineered strains Po1g KdHR and Po1g KlHR was carried out for 3 days in a $5 \mathrm{~L}$-fermenter. The titers of D-limonene and L-limonene were only $2.222 \mathrm{mg} / \mathrm{L}$ and $1.975 \mathrm{mg} / \mathrm{L}$, respectively (the productivities of $\mathrm{D}$-limonene and L-limonene were $0.0309 \mathrm{mg} / \mathrm{L} / \mathrm{h}$ and $0.0274 \mathrm{mg} / \mathrm{L} / \mathrm{h}$, respectively), and the titers of D-limonene and L-limonene further declined with increasing fermentation time. The low titers can possibly be attributed to non-optimal fed-batch medium and cultivation parameters (e.g., concentration of ions, $\mathrm{pH}$ and dissolved oxygen level) during the fed-batch fermentation of the engineered $Y$. lipolytica strains. Importantly, $n$-dodecane overlay for capturing volatile limonene, which played a critical role for improving limonene production in shake flasks, might not be beneficial in the fermenter. Particularly, the sparging used for aerating the culture in the fermenter increases air flow through the culture and can accelerate vaporization of limonene, thus leading to low titers; extensive reconfiguration will be required for alternative setups to trap limonene produced in the fermenter. Therefore, further optimization is required to overcome these hurdles.

\section{An investigation into the potential use of WCO as the carbon source for limonene production using the engineered $Y$. lipolytica strains}

WCO mainly refers to frying oil used at high temperatures, edible fat mixed in kitchen waste and oily waste water directly discharged into the sewer. And annual global production of WCO exceeds 29 million tons [62]. Especially, China is the biggest producer of WCO in the world, with 4.5 million tons of WCO produced each year. If this waste is improperly handled, it will pollute the cities, as well as flow back to the dining tables in case of weak government supervisions, profit-driven practices and lack of rapid testing methods. According to the statistics, China's WCO flowing back to the dining table amounts to 2.0-3.0 million tons, which poses a great health hazard to consumers [63]. Therefore, disposal and reutilization of WCO are long-term alarming problems in China. Disposal of WCO by appropriate management is thus vital $[64,65]$. Traditionally, WCO has been used for diesel engine fuel production, soap fabrication, as additives in domestic animal feedstock or as an inexpensive cleaning agent [26]. However, most recent research contributions on bioremediation of WCO has focused on the utilization of WCO as raw and economical feedstock for production of different valuable products. These strategies simultaneously degraded WCO and obtained high value-added products, which would be more attractive methods for disposal of WCO from both economic and environmental protection perspectives $[27,66-70]$. Y. lipolytica is capable of growing on various inexpensive carbon sources including alkanes, alkenes, fats, alcohols and organic acids. Owing to this unique ability of Y. lipolytica, we propose to investigate the potential use of WCO as the carbon source for limonene production from the engineered limonene-producing $Y$. lipolytica strains. To this end, Po1g KdHR and Po1g KlHR were cultured under different concentrations of $\mathrm{WCO}(0 \%, 10 \%, 30 \%, 50 \%$, and $70 \%$ ) as carbon source instead of glucose. Commercial vegetable oil was also used as a control (information on fatty acid composition of the commercial vegetable oil and waste cooking oil can be found in Additional file 1: Table S1). The cells were cultivated in $250-\mathrm{mL}$ shake flasks for 5 days using the optimum fermentation parameters determined afore, i.e., temperature of $20{ }^{\circ} \mathrm{C}$, rotation speed of $250 \mathrm{rpm}$ and $0.2 \%$ of $\mathrm{MgSO}_{4} \cdot 7 \mathrm{H}_{2} \mathrm{O}$. The results showed $\mathrm{D}$-limonene and L-limonene were successfully produced using WCO as sole carbon source, with the highest respective titers of $2.514 \mathrm{mg} / \mathrm{L}$ (Fig. 7a) and $2.723 \mathrm{mg} / \mathrm{L}$ (Fig. 7b) achieved with $70 \%$. These represent approximately $11 \%$ of $\mathrm{D}$-limonene and $16 \%$ of L-limonene increase in titers as compared to those obtained using glucose as carbon source and over 20-fold improvement compared with the initial titers of $0.124 \mathrm{mg} / \mathrm{L}$ D-limonene and $0.126 \mathrm{mg} / \mathrm{L}$ L-limonene before strain and fermentation optimization. Cell growth on WCO and vegetable oil was also monitored by determining the $\mathrm{OD}_{600}$ of Po1g KdHR and Po1g KlHR. With the same number of carbon units, the $\mathrm{OD}_{600}$ achieved by feeding $1.18 \%(\mathrm{w} / \mathrm{v}) \mathrm{WCO}$ as carbon source was significantly higher than that with $2 \%$ glucose (Additional file 1: Fig. S3), demonstrating that our engineered limonene-producing $Y$. lipolytica strains can grow very efficiently and robustly on WCO as the sole carbon source, and the higher yield of limonene from WCO and vegetable oil may be attributed to the higher biomass (Fig. 7). Taken together, we have developed Y. lipolytica strains and optimized fermentation conditions that can potentially serve as a platform for 


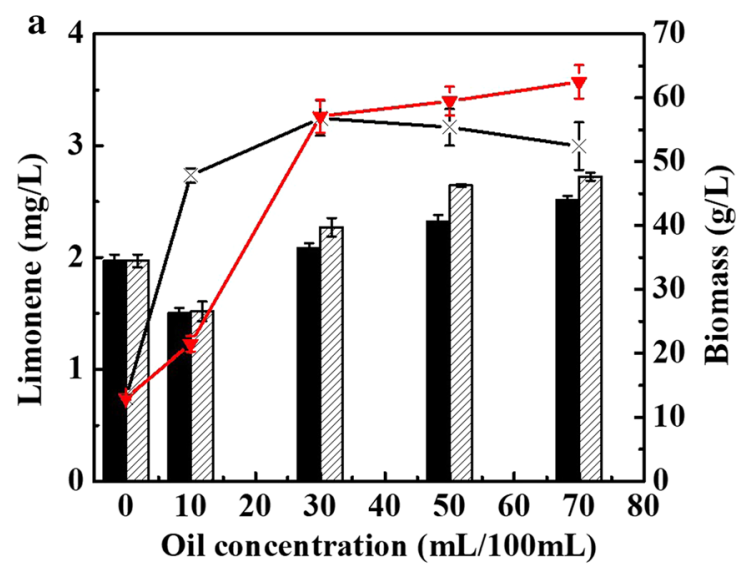

Po1g KdHR WCO Con $\longleftarrow$ Po1g KdHR WCO Bio
$\square$ Pold Po1g KdHR VO Con $\longrightarrow$ Po1g KdHR VO Bio

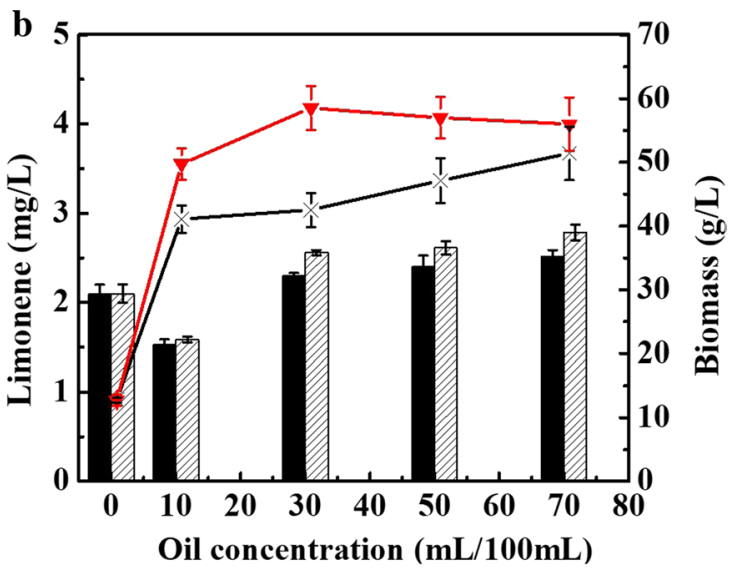

Po1g KIHR WCO Con $\longrightarrow$ Po1g KIHR WCO Bio Po1g KIHR VO Con $\longrightarrow$ Po1g KIHR VO Bio

Fig. 7 Effect of WCO on D-limonene or L-limonene accumulation in Po1g KdHR or Po1g KIHR. a Effect of WCO on D-limonene accumulation of Po1g KdHR. D-limonene accumulation of Po1g KdHR from WCO; Pllllls: D-limonene accumulation of Po1g KdHR from vegetable oil; $\longrightarrow$ biomass accumulation of Po1g KdHR from WCO; ——: biomass accumulation of Polg KdHR from vegetable oil. $\mathbf{b}$ Effect of WCO on L-limonene accumulation of Po1g KIHR. L L-limonene accumulation of Po1g KIHR from WCO; LIII) L-limonene accumulation of Po $1 \mathrm{~g}$ KIHR from vegetable oil; —— biomass accumulation of Po1g KIHR from WCO; $\longrightarrow$ : biomass accumulation of Po1g KIHR from vegetable oil. The cultivation of Po $1 \mathrm{~g} \mathrm{KdHR}$ or Po $1 \mathrm{~g} \mathrm{KIHR} \mathrm{was} \mathrm{performed} \mathrm{at} \mathrm{the} \mathrm{optimum} \mathrm{fermentation} \mathrm{temperature} \mathrm{of} 20^{\circ} \mathrm{C}$, the optimum rotation speed of $250 \mathrm{rpm}$, the optimum initial $\mathrm{OD}_{600}$ of 2.0, the optimum pH of 5.74 , the best $n$-dodecane volume $10 \%$ and the optimum $\mathrm{Mg}^{2+}$ concentration $0.2 \%$ of $\mathrm{MgSO}_{4} \cdot 7 \mathrm{H}_{2} \mathrm{O}$ in the optimal $50 \mathrm{~mL}$-final-volume of WCO medium in a 250 -mL shake flask for 5 days with different concentrations of WCO $(0 \%, 10 \%$, $30 \%, 50 \%$, and $70 \%$ ) as carbon source instead of glucose. Commercial vegetable oil was also used as a control. D-limonene accumulation of Po1g KdHR from WCO; biomass accumulation of Polg KIHR. All values presented are the mean of three biological replicates \pm standard deviation

sustainable and economical production of D-limonene and L-limonene from WCO.

\section{Conclusions}

There is a great demand for limonene due to its wide range of industrial applications. Metabolic engineering of microorganisms has provided a platform for effective production of these valuable products. Here, we constructed a new pathway in Y. lipolytica using metabolic engineering tools to enzymatically convert the abundant acetyl-CoA pool in the oleaginous yeast host to $\mathrm{D}$-limonene and $\mathrm{L}$-limonene. Combining metabolic engineering strategies with optimization of fermentation conditions and fed-batch fermentation in shake flasks, final titers of $11.705 \mathrm{mg} / \mathrm{L}(0.443 \mathrm{mg} / \mathrm{g})$ for D-limonene and $11.088 \mathrm{mg} / \mathrm{L}(0.385 \mathrm{mg} / \mathrm{g})$ for $\mathrm{L}$-limonene were achieved from glucose in this study. In addition, we also found that $\mathrm{Mg}^{2+}$ improved both the yields of D-limonene and L-limonene from the engineering yeast more than $\mathrm{Mn}^{2+}$. Importantly, we investigated the use of WCO to produce limonene and demonstrated that $\mathrm{WCO}$ is a superior substrate to glucose for producing limonene using the engineered limonene-producing $Y$. lipolytica strains. D-limonene and L-limonene were successfully produced at the respective titers of $2.514 \mathrm{mg} / \mathrm{L}$ and $2.723 \mathrm{mg} / \mathrm{L}$ with WCO as the carbon source. These are over 20-fold improvements compared to the initial titers of $0.124 \mathrm{mg} / \mathrm{L}$ D-limonene and $0.126 \mathrm{mg} / \mathrm{L} \mathrm{L}$-limonene before strain and fermentation optimization.

It is always beneficial that the biochemicals produced within cell factories are exported into the extracellular environment for collection in industrial applications. And the export of biochemicals has also been shown to successfully enhance production of target compounds. Although transport of hydrocarbons by passive diffusion or active transporters is known, the exact transport mechanism of limonene has not been reported [36, 37]. Therefore, we did not engineer the efflux/transportation of limonene in this study; much fundamental studies are required in future before this could be achieved. Furthermore, in this study, a dodecane overlay was used to extract limonene from the yeast culture because limonene could be trapped in a dodecane phase added to the medium during fermentation to enhance production level. Although this method is currently used widely for capturing volatile terpenes and other compounds produced by $Y$. lipolytica and different microbes, it may not be the best method for optimal harvest of limonene 
from this yeast, which exhibit alkane-binding behavior in large-scale production of limonene. In addition, the limonene production titers in this work are lower than that in previous reports (Table 1). The higher limonene productions were mostly achieved with $E$. coli but it is not the preferred host for industrial fermentation, particularly due to the susceptibility of $E$. coli to phage contamination [71]. Although higher limonene titer has been achieved in $Y$. lipolytica through extensive metabolic engineering [72], the unprecedented work reported here on optimizing fermentation conditions for limonene production has nevertheless provided valuable insights that will facilitate further efforts in the biosynthesis of this compound. In future, we will optimize the capture method for limonene from this yeast strain by evaluating the extraction efficiency of different methods [73], such as extraction with other solvents, solid-phase extraction and headspace trapping, along with the implementation of various engineering strategies to further improve the titer of limonene with our engineered Y. lipolytica strains.

In conclusion, we established that the oleaginous yeast strain Y. lipolytica is an attractive platform for providing a feasible and scalable route to limonene overproduction from WCO. Successful development of the Y. lipolytica platform to convert WCO into limonene will bring about a major breakthrough in the waste conversion and biochemical industries. However, the limonene downstream pathway was characterized in the present study. Given the accumulated knowledge about limonene downstream pathway and its regulation, it is believed that high productivity of limonene can be achieved by further strain engineering in our next experiments.

\section{Materials and methods}

All chemicals, solvents, and media components were purchased and used without modification, Pml I, Kpn I, Spe I and Nru I were purchased from New England Biolabs (Beijing, China), ClonExpress ${ }^{\circledR}$ II one step cloning kit, $2 \times$ Rapid taq master mix and $2 \times$ Phanta $^{\circledR}$ max master mix were purchased from Vazyme Biotech Co., Ltd. (Nanjing, China), tryptone and yeast extract were purchased from Thermo Scientific Oxoid Microbiology Products (Basingstoke, England), D-limonene and $n$-dodecane were purchased from Aladdin ${ }^{\circledR}$ (Shanghai, China), L-limonene were purchased from Tokyo Chemical Industry (Shanghai, China), and salmon sperm DNA, plasmid extraction mini kit and DNA purification kit were purchased from Solarbio Life Sciences (Beijing, China). Escherichia coli DH5 $\alpha$ was used for plasmid construction and amplification and $E$. coli strains were routinely cultured in LB medium supplemented with $100 \mu \mathrm{g} / \mathrm{mL}$ of ampicillin at $37{ }^{\circ} \mathrm{C}$. $Y$. lipolytica Polg KU70 $\Delta$ was used as the base strain in this
Table 2 Plasmids used in this study

\begin{tabular}{|c|c|c|}
\hline Plasmid & Features & Reference \\
\hline pYLEX1 & 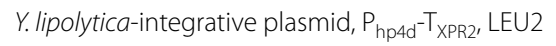 & {$[78]$} \\
\hline pYLdLS & $P_{\text {hp4d }}$ dLS-T $_{\text {XPR2, }}$ LEU2 & This study \\
\hline pYLILS & $P_{\text {hp4d }}-{ }^{-I L S-T}$ XPR2, LEU2 & This study \\
\hline pYLA1 & $P_{\text {hp4d }}-A 1-T_{X P R 2}, L E U 2$ & This study \\
\hline pYLpA2 & $P_{\text {hp4d }}-$ PA2-T XPR2, LEU2 & This study \\
\hline pYLA2 & $P_{\text {hp } 4 d^{-}}-A 2-T_{X P R 2}$, LEU2 & This study \\
\hline pYLHS & $P_{\text {hp4d }}-\mathrm{HS}-T_{X P R 2}$, LEU2 & This study \\
\hline pYLHR & $P_{\text {hp } 4 d}-H R-T_{X P R 2}$, LEU2 & This study \\
\hline pYLMK & $P_{\text {hP4d }}-M K-T_{X P R 2}, L E U 2$ & This study \\
\hline pYLPK & $P_{\text {hp4d }}-P K-T_{X P R 2}$, LEU2 & This study \\
\hline pYLPD & $P_{\text {hp4d }}-P D-T_{X P R 2}, L E U 2$ & This study \\
\hline pYLIDI & $P_{\text {hp4d }}-1$ DI-T XPR2, LEU2 & This study \\
\hline pYLGS & $P_{\text {hp4d }}{ }^{-G S-T}$ XPR2, LEU2 & This study \\
\hline pYLFS & $P_{\text {hp } 4 d}-$ FS-T XPR2, LEU2 & This study \\
\hline pYLdA1 & $P_{\text {hp4d }}-d L S-T_{X P R 2}, P_{h p 4 d}-A 1-T_{X P R 2}$, LEU2 & This study \\
\hline PYLdA2 & $P_{\text {hp } 4 d}-d L S-T_{X P R 2}, P_{h p 4 d}-A 2-T_{X P R 2}$, LEU2 & This study \\
\hline pYLdHS & $P_{h p 4 d}-d L S-T_{X P R 2}, P_{h p 4 d}-H S-T_{X P R 2}, L E U 2$ & This study \\
\hline pYLdHR & $P_{\text {hp4d }}-d L S-T_{X P R 2}, P_{\text {hp4d }}-{ }^{-H R-T} T_{X P R 2}$, LEU2 & This study \\
\hline pYLdMK & $P_{\text {hp } 4 d}-d L S-T_{X P R 2}, P_{\text {hp4d }}-M K-T_{X P R 2}$ LEU2 & This study \\
\hline pYLdPK & $P_{\text {hp } 4 d}-d L S-T_{X P R 2}, P_{\text {hp } 4 d}-{ }^{-P K-T}$ XPR2, LEU2 & This study \\
\hline pYLdPD & $P_{\text {hp } 4 d}-d L S-T_{X P R 2}, P_{\text {hp4d }}-$ PD-T XPR2, LEU2 & This study \\
\hline pYLdIDI & $P_{\text {hp4d }}-d L S-T_{X P R 2}, P_{\text {hp4d }}-1 D \mid-T_{X P R 2}, L E U 2$ & This study \\
\hline pYLdGS & $P_{h p 4 d}-d L S-T_{X P R 2}, P_{h p 4 d}-G S-T_{X P R 2}, L E U 2$ & This study \\
\hline pYLdFS & $P_{h p 4 d}-d L S-T_{X P R 2}, P_{h p 4 d}-F S-T_{X P R 2}, L E U 2$ & This study \\
\hline PYLIA1 & $P_{\text {hp } 4 d^{-}}-1 L S-T_{X P R 2}, P_{h p 4 d}-A 1-T_{X P R 2}, L E U 2$ & This study \\
\hline PYLIA2 & $P_{\text {hp } 4 d}-1 L S-T_{X P R 2}, P_{h p 4 d}-A 2-T_{X P R 2}, L E U 2$ & This study \\
\hline pYLIHS & 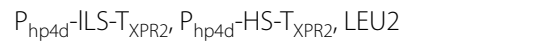 & This study \\
\hline pYLIHR & $P_{\text {hp } 4 d}-1 L S-T_{X P R 2}, P_{h p 4 d}-H R-T_{X P R 2}, L E U 2$ & This study \\
\hline pYLIMK & $P_{\text {hp } 4 d^{-1 L S}-T_{X P R 2}} P_{\text {hp4d }}-M K-T_{X P R 2}$, LEU2 & This study \\
\hline pYLIPK & $P_{\text {hp } 4 d^{-}}-1 L S-T_{X P R 2}, P_{h p 4 d}-P K-T_{X P R 2}, L E U 2$ & This study \\
\hline pYLIPD & $P_{\text {hp } 4 d^{-}-1 L S-T_{X P R 2}}, P_{h p 4 d}-P D-T_{X P R 2}, L E U 2$ & This study \\
\hline pYLIIDI & $P_{\text {hp4d }}{ }^{-1 L S-T_{X P R 2}}, P_{h p 4 d}-1 D I-T_{X P R 2}, L E U 2$ & This study \\
\hline pYLIGS & $P_{\text {hp4d }}{ }^{-I L S-T_{X P R 2}}, P_{h p 4 d}-G S-T_{X P R 2}, L E U 2$ & This study \\
\hline pYLIFS & $P_{\text {hp4d }}-1 L S-T_{X P R 2}, P_{h p 4 d}-{ }^{-F S-T}$ XPR2, LEU2 & This study \\
\hline
\end{tabular}

study. Routine cultivation of $Y$. lipolytica strains was carried out at $30{ }^{\circ} \mathrm{C}$ in YPD medium (yeast extract, $10 \mathrm{~g} / \mathrm{L}$; peptone, $20 \mathrm{~g} / \mathrm{L}$; glucose, $20 \mathrm{~g} / \mathrm{L}$ ). In experiments employing $\mathrm{WCO}$ as the carbon source, $2 \%$ glucose was removed and replaced with appropriate concentrations of WCO. WCO was collected from a local kitchen; vegetable oil was obtained commercially (Luhua brand). The growth medium was also supplemented with Tween- 80 when WCO was used as the carbon source in WCO medium. All of the recombinant plasmids were constructed using the One Step Cloning Kit from Vazyme Biotech Co., Ltd (Nanjing, China). Plasmids used in this study are listed in Table 2 and strains are listed in Table 3. PCR primers 
Table 3 Strains used in this study

\begin{tabular}{|c|c|c|}
\hline Strains & Genotype & Reference \\
\hline Po1g KU70 & MatA, leu2-270, ura3-302::URA3, xpr2-332, axp-2, ku70- & [74] \\
\hline Po1g KdLS & MatA, leu2-270, ura3-302::URA3, xpr2-332, axp-2, ku70-, dLS & This study \\
\hline Po1g KILS & MatA, leu2-270, ura3-302::URA3, xpr2-332, axp-2, ku70-, ILS & This study \\
\hline Po1g KdA1 & MatA, leu2-270, ura3-302::URA3, xpr2-332, axp-2, ku70-, dLS, ACOAAT1 & This study \\
\hline Po1g KdA2 & MatA, leu2-270, ura3-302::URA3, xpr2-332, axp-2, ku70-, dLS, ACOAAT2 & This study \\
\hline Polg KdHS & MatA, leu2-270, ura3-302::URA3, xpr2-332, axp-2, ku70-, dLS, HMGS & This study \\
\hline Polg KdHR & MatA, leu2-270, ura3-302::URA3, xpr2-332, axp-2, ku70-, dLS, HMGR & This study \\
\hline Po1g KdMK & MatA, leu2-270, ura3-302::URA3, xpr2-332, axp-2, ku70-, dLS, MK & This study \\
\hline Polg KdPK & MatA, leu2-270, ura3-302::URA3, xpr2-332, axp-2, ku70-, dLS, PMK & This study \\
\hline Polg KdPD & MatA, leu2-270, ura3-302::URA3, xpr2-332, axp-2, ku70-, dLS, PMVADO & This study \\
\hline Po1g KdIDI & MatA, leu2-270, ura3-302::URA3, xpr2-332, axp-2, ku70-, dLS, IPPDI & This study \\
\hline Po1g KdGS & MatA, leu2-270, ura3-302::URA3, xpr2-332, axp-2, ku70-, dLS, GGPPS & This study \\
\hline Polg KdFS & MatA, leu2-270, ura3-302::URA3, xpr2-332, axp-2, ku70-, dLS, FPPS & This study \\
\hline Po1g KIA1 & MatA, leu2-270, ura3-302::URA3, xpr2-332, axp-2, ku70-, ILS, ACOAAT1 & This study \\
\hline Po1g KIA2 & MatA, leu2-270, ura3-302::URA3, xpr2-332, axp-2, ku70-, ILS, ACOAAT2 & This study \\
\hline Polg KIHS & MatA, leu2-270, ura3-302::URA3, xpr2-332, axp-2, ku70-, ILS, HMGS & This study \\
\hline Polg KIHR & MatA, leu2-270, ura3-302::URA3, xpr2-332, axp-2, ku70-, ILS, HMGR & This study \\
\hline Polg KIMK & MatA, leu2-270, ura3-302::URA3, xpr2-332, axp-2, ku70-, ILS, MK & This study \\
\hline Po1g KIPK & MatA, leu2-270, ura3-302::URA3, xpr2-332, axp-2, ku70-, ILS, PMK & This study \\
\hline Polg KIPD & MatA, leu2-270, ura3-302::URA3, xpr2-332, axp-2, ku70-, ILS, PMVADO & This study \\
\hline Po1g KIIDI & MatA, leu2-270, ura3-302::URA3, xpr2-332, axp-2, ku70-, ILS, IPPDI & This study \\
\hline Polg KIGS & MatA, leu2-270, ura3-302::URA3, xpr2-332, axp-2, ku70-, ILS, GGPPS & This study \\
\hline Po1g KIFS & MatA, leu2-270, ura3-302::URA3, xpr2-332, axp-2, ku70-, ILS, FPPS & This study \\
\hline
\end{tabular}

used in this study were synthesized by Genewiz (Jiangsu, China) and are listed in Additional file 1: Table S2.

\section{Plasmid construction}

The D-limonene synthase gene ( $d L S$, GenBank ID: AF514289) from C. limon and L-limonene synthase gene (lLS, GenBank ID: L13459) from M. spicata were synthesized and codon-optimized by Genewiz (Jiangsu, China). The sequences encoding the transit peptide within both genes were removed according to the previous literatures $[27,28]$. The genes $d L S$ and $l L S$ were cloned into pYLEX1 (Additional file 1: Fig. S13) with primers dLS-F/dLS-R and ILS-F/ILS-R (Additional file 1: Table S2) to yield plasmid pYLdLS and pYLILS (Additional file 1: Fig. S14), respectively. The intron-containing gene, pACOAAT2, was cloned into pYLEX1 with primers pACOAAT2-1-F/ pACOAAT2-1-R and pACOAAT2-2-F/pACOAAT2$2-R$ to remove intron and yield plasmid pYLpA2, the gene ACOAAT2 was amplified from plasmid pYLpA2 and cloned into pYLEX1 with primers ACOAAT2-F/ pACOAAT2-2-R to yield plasmid pYLA2. The genes
ACOAAT1, HMGS, HMGR, MK, PMK, PMVADO, IPPDI, GGPPS, and FPPS were cloned into pYLEX1 with primers ACOAAT1-F/ACOAAT1-R, HMGS-F/HMGSR, HMGR-F/HMGR-R, MK-F/MK-R, PMK-F/PMK-R, PMVADO-F/PMVADO-R, IPPDI-F/IPPDI-R, GGPPSF/GGPPS-R, FPPS/FPPS-R to yield plasmid pYLA1, pYLHS, pYLHR, pYLMK, pYLPK, pYLPD, pYLIDI, pYLGS and pYLFS, respectively. The expression cassettes of ACOAAT1, ACOAAT2, HMGS, HMGR, MK, PMK, PMVADO, IPPDI, GGPPS and FPPS were cloned into pYLdLS and pYLlLS with primers BDH-F/BDH$\mathrm{R}, \mathrm{BDH} 2-\mathrm{F} / \mathrm{BDH} 2-\mathrm{R}$ and FSBDH-F/BDH2-R to yield plasmid pYLdA1, pYLlA1, pYLdA2, pYLlA2, pYLdHS, pYLlHS, pYLdHR, pYLlHR, pYLdMK, pYLIMK, pYLdPK, pYLIPK, pYLdPD, pYLIPD, pYLdIDI, pYLIIDI, pYLdGS, pYLIGS, pYLdFS and pYLlFS (Additional file 1: Fig. S15), respectively. All plasmids were first linearized with Not I or Spe I and then transformed into competent cells of $Y$. lipolytica strains using the lithium acetate/singlestranded carrier DNA/polyethylene glycol method [74]. 


\section{Strain construction}

Yeast colonies of $Y$. lipolytica Po1g KU70 $\Delta$ were grown in $30 \mathrm{~mL}$ of fresh YPD medium for $24 \mathrm{~h}$. Cells were pelleted and washed twice with $20 \mathrm{~mL}$ Tris-EDTA (TE) buffer (10 mM Tris, $1 \mathrm{mM}$ EDTA, pH 7.5) and once with $0.1 \mathrm{M}$ lithium acetate $(\mathrm{pH}$ 6.0). Then resuspend the cell with $3 \mathrm{~mL}$ of $0.1 \mathrm{M}$ lithium acetate ( $\mathrm{pH} 6.0)$, and incubate for $10 \mathrm{~min}$ at room temperature and aliquot $100 \mu \mathrm{L}$ into sterile $1.5 \mathrm{~mL}$ tubes. The competent cells were then mixed by vortexing with $0.7 \mathrm{~mL}$ of $40 \%$ PEG-4000, $10 \mu \mathrm{L}$ of denatured salmon sperm DNA and $10 \mu \mathrm{L}$ of linearized recombination plasmids for $1 \mathrm{~h}$. The transformation mixture and incubated at $39^{\circ} \mathrm{C}$ for $1 \mathrm{~h}$. Then add $1 \mathrm{~mL}$ YPD medium and recover for $2 \mathrm{~h}$ at $30{ }^{\circ} \mathrm{C}$ and $225 \mathrm{rpm}$. Following that, the transformation mixture was pelleted, resuspended in water and plated directly onto YNB plates. After selection, the following engineered Y. lipolytica strains were generated: Po1g KdLS, Po1g KILS, Po1g KdA1, Po1g K1A1, Po1g KdA2, Po1g K1A2, Po1g KdHS, Po1g KlHS, Po1g KdHR, Po1g KlHR, Po1g KdMK, Po1g KlMK, Po1g KdPK, Po1g KlPK, Po1g KdPD, Po1g KlPD, Po1g KdIDI, Po1g KlIDI, Po1g KdGS, Po1g KlGS, Po1g KdFS and Po1g KIFS.

\section{Culturing the engineered $Y$. lipolytica strains for $D$-limonene and L-limonene production}

To produce D-limonene and L-limonene, seed cultures were prepared by inoculating $5 \mathrm{~mL}$ of YPD medium in the 20-mL culture tubes with the engineered $Y$. lipolytica strains. The cells were grown at $30{ }^{\circ} \mathrm{C}$ for $24 \mathrm{~h}$ with agitation. Following that, $250-\mathrm{mL}$ flasks containing $30 \mathrm{~mL}$ of YPD medium were inoculated to $\mathrm{OD}_{600} 0.1$ with the seed cultures. All cultures were shaken at $200 \mathrm{rpm}$ and $30{ }^{\circ} \mathrm{C}$. To avoid loss of limonene during cultivation, $8 \%$ of $n$-dodecane overlay was added into the YPD medium prior to cultivation. Samples were then collected at day 5 . To improve yields of D-limonene and L-limonene further, the effect of cultivation conditions was investigated, such as temperature, rotation speed, $\mathrm{pH}$, the volume of culture medium, the volume of $n$-dodecane, initial $\mathrm{OD}_{600}$ and $\mathrm{Mg}^{2+}$ concentration. An example of optimization of temperature is given below, the cells were cultivated $200 \mathrm{rpm}$ with an initial $\mathrm{OD}_{600}$ of 0.1 and $8 \%$ of $n$-dodecane in $30 \mathrm{~mL}$ of liquid YPD medium in a $250-\mathrm{mL}$ shake flask for 5 days at various cultivation temperatures, i.e., 15, 20, 25 and $30{ }^{\circ} \mathrm{C}$. Finally, different concentrations of WCO and vegetable oil $(0 \%, 10 \%, 30 \%, 50 \%$, and $70 \%)$ were added as carbon sources instead of glucose in YPD medium using the optimum fermentation parameters determined afore, and the dodecane layer was added into WCO medium before fermentation. At the end of fermentation, the mixture of the dodecane layer and WCO formed a onelayer system after centrifuging at $7500 \mathrm{rpm}$ for $10 \mathrm{~min}$. Therefore, the upper one-layer organic phase was used for analysis of limonene production by GC/MS.

\section{Fed-batch cultures}

Fed-batch cultures were conducted in YPDM medium (yeast extract, $10 \mathrm{~g} / \mathrm{L}$; peptone, $20 \mathrm{~g} / \mathrm{L}$; glucose, $20 \mathrm{~g} / \mathrm{L}$; $\mathrm{MgSO}_{4} \cdot 7 \mathrm{H}_{2} \mathrm{O}, 2 \mathrm{~g} / \mathrm{L}$ ). Overnight cultures of the engineered strains were diluted in $50 \mathrm{~mL}$ YPD to $\mathrm{OD}_{600} 2.0$ in $250 \mathrm{~mL}$ shake flasks. Intermittent feeding with glucose was conducted at 24-h intervals, with $1 \mathrm{~mL}$ of $50 \%$ glucose being added at each feeding time-point. In the 5 L-bioreactor, the initial volume of YPDM medium was $2 \mathrm{~L}$ and continuous feeding was conducted, with $120 \mathrm{~mL}$ of $50 \%$ glucose fed at a constant rate over every 24-h cultivation period from $10 \mathrm{~h}$ to $72 \mathrm{~h} ; 155 \mathrm{~g}$ glucose was fed in total. The $\mathrm{pH}$ value, temperature and oxygen saturation were fixed at 5.7 (regulated with $20 \%$ $\mathrm{NaOH}$ and $40 \%$ citric acid), $28{ }^{\circ} \mathrm{C}$, and $20 \%$ (adjusted by stirring and aeration), respectively. AntiFoam B-396 (Guangdong Zhong Lian Bang Fine Chemical Co., Ltd, China) was used as the defoaming agent. Then, $5 \mathrm{~mL}$ of wet cell culture was harvested and washed twice with distilled water. The biomass was dried at $105{ }^{\circ} \mathrm{C}$ until the weight was stable.

\section{GC-MS analysis of D-limonene and L-limonene produced in the engineered $Y$. lipolytica strains}

For the determination of D-limonene and L-limonene, all cultures were centrifuged at $7500 \mathrm{rpm}$ for $10 \mathrm{~min}$ at each time of sampling. After fermentation, the volumes of $n$-dodecane phase were average $96 \%$ left compared to the initial volumes of $n$-dodecane. Specially, in the experiments using $\mathrm{WCO}$ as the sole carbon source, this volume of organic phase analyzed by GC-MS represents the total volume of $\mathrm{WCO}$ and $n$-dodecane. Then, $1 \mu \mathrm{L}$ organic phase was then analyzed by GC-MS using an Agilent 7890A GC with an 5975C MSD equipped with a HP-5MS column $(30 \mathrm{~m} \times 0.25 \mathrm{~mm} \times 0.25 \mu \mathrm{m}$, Agilent, Santa Clara, CA, USA). GC oven temperature was initially held at $60^{\circ} \mathrm{C}$ for $2 \mathrm{~min}$, and then ramped to $140{ }^{\circ} \mathrm{C}$ at a rate of $5{ }^{\circ} \mathrm{C} / \mathrm{min}$. It was then subsequently ramped at $10{ }^{\circ} \mathrm{C} / \mathrm{min}$ to $280{ }^{\circ} \mathrm{C}$ and held for $5 \mathrm{~min}$. The split ratio was 10:1. Helium was used as the carrier gas, with an inlet pressure of 13.8 psi. The injector was maintained at $280^{\circ} \mathrm{C}$ and the ion source temperature was set to $230{ }^{\circ} \mathrm{C}$. Final data analysis was achieved using Enhanced Data Analysis software (Agilent, Santa Clara, CA, USA). 


\section{Supplementary information}

Supplementary information accompanies this paper at https://doi. org/10.1186/s13068-019-1580-y.

Additional file 1: Table S1. Information on fatty acid composition of the commercial vegetable oil and waste cooking oil. Table S2. Primers used in PCR. Figure S1. Inhibitory effects of $d$-limonene and L-limonene on S. cerevisiae AY15 and Y. lipolytica Po1g KU70 $\triangle$. Figure S2. GC-MS analysis of solvent overlay-extracted limonene from engineered strains cultures. Figure S3. Effect of different carbon sources on growth of Y. lipolytica. Figure S4. Effect of addition of glucose on biosynthesis of limonene. Figure S5. The $\mathrm{OD}_{600}$ values of different engineered Y. lipolytica strains cultured in YPD medium. Figure S6. Effect of temperature on volatilization of limonene. Figure S7. The pHs during fermentation of the engineered Y. lipolytica Po1g KdHR and Po1g KIHR strains in a 250-mL shake flask. Figure S8. The data of limonene batch-fermentation parameters in the 5 L-fermenter. Figure S9. Effect of $\mathrm{Mg}^{2+}$ on growth of $Y$. lipolytica. Figure S10. Effect of $\mathrm{Mn}^{2+}$ on D-limonene or L-limonene accumulation in Polg KdHR or Polg KIHR. Figure S11. Effect of concentration of peptone on the production of D-limonene and L-limonene. Figure S12. The data of limonene fed-batch fermentation parameters in the 5- $L$ fermenter. Figure S13. Map of the plasmid pYLEX1. Figure S14. Map of the recombinant plasmid pYLdLS. Figure S15. Maps of the overexpression recombinant plasmid pYLA1 and pYLdA1. Figure S16. The GC-MS analysis of fatty acid composition in waste cooking oil and commercial vegetable oil.

\section{Abbreviations}

GC/MS: Gas chromatography/mass spectrometry; $\mathrm{OD}_{600}$ : Optical density at $600 \mathrm{~nm}$; LB medium: 0.5\% yeast extract, 1\% tryptone and 1\% $\mathrm{NaCl}$; YPD medium: $1 \%$ yeast extract, $2 \%$ peptone and $2 \%$ glucose; WCO medium: $1 \%$ yeast extract, $2 \%$ peptone and appropriate concentration of WCO; YNB plate: $2 \%$ glucose, $0.67 \%$ yeast nitrogen base without amino acids and $2 \%$ agar; PCR: Polymerase chain reaction; DCW: Dry cell weight.

\section{Acknowledgements}

This work was supported by the Research Foundation of Tianjin Municipal Education Commission, China (2017ZD03), the Natural Science Foundation of Tianjin, China (17JCYBJC40800), the Innovative Research Team of Tianjin Municipal Education Commission, China (TD13-5013), Public Service Platform Project for Selection and Fermentation Technology of Industrial Microorganisms, China (17PTGCCX00190), Tianjin Municipal Science and Technology Project (18PTSYJC00140), the Open Fund of Ministry of Education Key Laboratory of Molecular Microbiology and Technology, Nankai University, Startup Fund for "Haihe Young Scholars" of Tianjin University of Science and Technology, the Thousand Young Talents Program of Tianjin, China.

\section{Authors' contributions}

AQY, DGX, and CYZ conceived and designed the study. YRP, YKZ, SLL, YZ, $\mathrm{J}$, and $\mathrm{ZHH}$ performed plasmid and strain construction, and fermentation experiments. YRP, YKZ, and ZHH wrote the manuscript. AQY, DGX, and CYZ revised the manuscript. All authors read and approved the final manuscript.

\section{Funding}

The Research Foundation of Tianjin Municipal Education Commission, China (2017ZD03), the Natural Science Foundation of Tianjin, China (17JCYBJC40800), the Innovative Research Team of Tianjin Municipal Education Commission, China (TD13-5013), Public Service Platform Project for Selection and Fermentation Technology of Industrial Microorganisms, China (17PTGCCX00190), Tianjin Municipal Science and Technology Project (18PTSYJC00140), the Open Fund of Ministry of Education Key Laboratory of Molecular Microbiology and Technology, Nankai University, Startup Fund for "Haihe Young Scholars" of Tianjin University of Science and Technology, the Thousand Young Talents Program of Tianjin, China.

\section{Availability of data and materials}

All relevant data generated or analyzed during this study were included in this published article.
Ethics approval and consent to participate

This manuscript does not contain any studies with human participants or animals performed by any of the authors.

\section{Consent for publication}

All authors give consent to publish the research in Biotechnology for Biofuels.

\section{Competing interests}

The authors declare that they have no competing interests.

Received: 8 November 2018 Accepted: 25 September 2019

Published online: 08 October 2019

\section{References}

1. Pakdel H, Pantea DM, Roy C. Production of DL-limonene by vacuum pyrolysis of used tires. J Anal Appl Pyrolysis. 2001;57(1):91-107.

2. Ciriminna R, Lomeli-Rodriguez M, Cara PD, Lopez-Sanchez JA, Pagliaro M. Limonene: a versatile chemical of the bioeconomy. Chem Commun. 2014;50(97):15288-96.

3. Kim YW, Kim MJ, Chung BY, Bang DY, Lim SK, Choi SM, et al. Safety evaluation and risk assessment of D-limonene. J Toxicol Environ Health B Crit Rev. 2013;16(1):17-38.

4. Karlberg AT, Magnusson K, Nilsson U. Air oxidation of D-limonene (the citrus solvent) creates potent allergens. Contact Dermatitis. 1992;26(5):332-40.

5. Wu F, Jacobs D, Mitchell C, Miller D, Karol MH. Improving indoor environmental quality for public health: impediments and policy recommendations. Environ Health Perspect. 2007;115(6):953-7.

6. Croteau RB, Davis EM, Ringer KL, Wildung MR. (-)-Menthol biosynthesis and molecular genetics. Naturwissenschaften. 2005;92(12):562-77.

7. Lin P-C, Saha R, Zhang F, Pakrasi HB. Metabolic engineering of the pentose phosphate pathway for enhanced limonene production in the cyanobacterium Synechocystis sp PCC 6803. Sci Rep. 2017;7:17503.

8. Chubukov V, Mingardon F, Schackwitz W, Baidoo EEK, Alonso-Gutierrez J, Hu Q, et al. Acute limonene toxicity in Escherichia coli is caused by limonene hydroperoxide and alleviated by a point mutation in alkyl hydroperoxidase AhpC. Appl Environ Microbiol. 2015;81(14):4690-6.

9. Du FL, Yu HL, Xu JH, Li CX. Enhanced limonene production by optimizing the expression of limonene biosynthesis and MEP pathway genes in E. coli. Bioresour Bioprocess. 2014;1(1):10.

10. Singh P, Shukla R, Prakash B, Kumar A, Singh S, Mishra PK, et al. Chemical profile, antifungal, antiaflatoxigenic and antioxidant activity of Citrus maxima Burm. and Citrus sinensis (L.) Osbeck essential oils and their cyclic monoterpene, DL-limonene. Food Chem Toxicol. 2010;48(6):1734-40.

11. Lopresto CG, Petrillo F, Casazza AA, Aliakbarian B, Perego P, Calabro V. A non-conventional method to extract D-limonene from waste lemon peels and comparison with traditional Soxhlet extraction. Sep Purif Technol. 2014;137:13-20.

12. Madzak C, Beckerich JM. Heterologous protein expression and secretion in Yarrowia lipolytica. In: Barth G, editor. Yarrowia lipolytica. Berlin Heidelberg: Springer; 2013. p. 1-76.

13. Abdel-Mawgoud AM, Markham KA, Palmer CM, Liu N, Stephanopoulos G, Alper HS. Metabolic engineering in the host Yarrowia lipolytica. Metab Eng. 2018. https://doi.org/10.1016/j.ymben.2018.07.016.

14. Beopoulos A, Cescut J, Haddouche R, Uribelarrea JL, Molina-Jouve C, Nicaud JM. Yarrowia lipolytica as a model for bio-oil production. Prog Lipid Res. 2009;48(6):375-87.

15. Madzak C, Gaillardin C, Beckerich JM. Heterologous protein expression and secretion in the non-conventional yeast Yarrowia lipolytica: a review. J Biotechnol. 2004;109(1-2):63-81.

16. Degenhardt J, Koellner TG, Gershenzon J. Monoterpene and sesquiterpene synthases and the origin of terpene skeletal diversity in plants. Phytochemistry. 2009;70(15-16):1621-37.

17. Ajikumar PK, Xiao WH, Tyo KEJ, Wang Y, Simeon F, Leonard E, et al. Isoprenoid pathway optimization for taxol precursor overproduction in Escherichia coli. Science. 2010;330(6000):70-4 
18. Martin VJJ, Pitera DJ, Withers ST, Newman JD, Keasling JD. Engineering a mevalonate pathway in Escherichia coli for production of terpenoids. Nat Biotechnol. 2003;21 (7):796-802.

19. Dobrowolski A, Mituła P, Rymowicz W, Mironczuk AM. Efficient conversion of crude glycerol from various industrial wastes into single cell oil by yeast Yarrowia lipolytica. Bioresour Technol. 2016;207:237-43.

20. Iwama R, Kobayashi S, Ohta A, Horiuchi H, Fukuda R. Fatty aldehyde dehydrogenase multigene family involved in the assimilation of n-alkanes in Yarrowia lipolytica. J Biol Chem. 2014;289(48):33275-86.

21. Iwama R, Kobayashi S, Ohta A, Horiuchi H, Fukuda R. Alcohol dehydrogenases and an alcohol oxidase involved in the assimilation of exogenous fatty alcohols in Yarrowia lipolytica. FEMS Yeast Res. 2015;15(3):fov014.

22. Kamzolova SV, Allayarov RK, Lunina JN, Morgunov IG. The effect of oxalic and itaconic acids on threo-Ds-isocitric acid production from rapeseed oil by Yarrowia lipolytica. Bioresour Technol. 2016;206:128-33.

23. Mironczuk AM, Rzechonek DA, Biegalska A, Rakicka M, Dobrowolski A. A novel strain of Yarrowia lipolytica as a platform for value-added product synthesis from glycerol. Biotechnol Biofuels. 2016;9(1):180.

24. Thevenieau F, Beopoulos A, Desfougeres T, Sabirova J, Albertin K, Zinjarde $\mathrm{S}$, et al. Uptake and assimilation of hydrophobic substrates by the oleaginous yeast Yarrowia lipolytica. In: Timmis KN, editor. Handbook of hydrocarbon and lipid microbiology. Heidelberg: Springer; 2010. p. 1513-27.

25. Oh YS, Maeng J, Kim SJ. Use of microorganism-immobilized polyurethane foams to absorb and degrade oil on water surface. Appl Microbiol Biotechnol. 2000;54(3):418-23.

26. Dominguez A, Deive FJ, Angeles Sanroman M, Longo MA. Biodegradation and utilization of waste cooking oil by Yarrowia lipolytica CECT 1240. Eur J Lipid Sci Technol. 2010;112(11):1200-8.

27. Katre G, Ajmera N, Zinjarde S, RaviKumar A. Mutants of Yarrowia lipolytica NCIM 3589 grown on waste cooking oil as a biofactory for biodiesel production. Microb Cell Fact. 2017;16:176.

28. Alonso-Gutierrez J, Chan R, Batth TS, Adams PD, Keasling JD, Petzold CJ, et al. Metabolic engineering of Escherichia coli for limonene and perillyl alcohol production. Metab Eng. 2013;19:33-41.

29. Colby SM, Alonso WR, Katahira EJ, McGarvey DJ, Croteau R. 4S-limonene synthase from the oil glands of spearmint (Mentha spicata). cDNA isolation, characterization, and bacterial expression of the catalytically active monoterpene cyclase. J Biol Chem. 1993;268(31):23016-24.

30. Davies FK, Work VH, Beliaev AS, Posewitz MC. Engineering limonene and bisabolene production in wild type and a glycogen-deficient mutant of Synechococcus sp. PCC 7002. Front Bioeng Biotechnol. 2014;2:1-11.

31. Lucker J, El Tamer MK, Schwab W, Verstappen FWA, van der Plas LHW, Bouwmeester $\mathrm{HJ}$, et al. Monoterpene biosynthesis in lemon (Citrus limon) — CDNA isolation and functional analysis of four monoterpene synthases. Eur J Biochem. 2002;269(13):3160-71.

32. Peng B, Nielsen LK, Kampranis SC, Vickers CE. Engineered protein degradation of farnesyl pyrophosphate synthase is an effective regulatory mechanism to increase monoterpene production in Saccharomyces cerevisiae. Metab Eng. 2018;47:83-93.

33. Jongedijk E, Cankar K, Ranzijn J, van der Krol S, Bouwmeester H, Beekwilder J. Capturing of themonoterpene olefin limonene produced in Saccharomyces cerevisiae. Yeast. 2015;32(1):159-71.

34. Vickers CE, Bongers M, Bydder SF, Chrysanthopoulos P, Hodson MP. Protocols for the production and analysis of isoprenoids in bacteria and yeast. In: McGenity T, Timmis K, Nogales Fernández B, editors. Hydrocarbon and lipid microbiology protocols. Berlin: Springer; 2015. p. 23-52.

35. Willrodt C, David C, Cornelissen S, Bühler B, Julsing MK, Schmid A. Engineering the productivity of recombinant Escherichia coli for limonene formation from glycerol in minimal media. Biotechnol J. 2014;9(8):1000-12.

36. Chen B, Ling H, Chang MW. Transporter engineering for improved tolerance against alkane biofuels in Saccharomyces cerevisiae. Biotechnol Biofuels. 2013;6(1):21.

37. Liu HH, Ji XJ, Huang H. Biotechnological applications of Yarrowia lipolytica: past, present and future. Biotechnol Adv. 2015;33(8):1522-46.

38. Thomsson E, Larsson C, Albers E, Nilsson A, Franzén CJ, Gustafsson L. Carbon starvation can induce energy deprivation and loss of fermentative capacity in Saccharomyces cerevisiae. Appl Environ Microbiol. 2003:69(6):3251-7.

39. Duetz WA, Bouwmeester $H$, van Beilen JB, Witholt B. Biotransformation of limonene by bacteria, fungi, yeasts, and plants. Appl Microbiol Biotechnol. 2003;61(4):269-77.
40. Ferrara MA, Almeida DS, Siani AC, Lucchetti L, Lacerda PS, Freitas A, et al. Bioconversion of $R-(+)$-limonene to perillic acid by the yeast Yarrowia lipolytica. Braz J Microbiol. 2013;44(4):1075-80.

41. van Rensburg E, Moleleki N, van der Walt JP, Botes PJ, van Dyk MS. Biotransformation of $(+)$ limonene and ( - )piperitone by yeasts and yeastlike fungi. Biotechnol Lett. 1997;19(8):779-82.

42. Malac J, Urbankova E, Sigler K, Gaskova D. Activity of yeast multidrug resistance pumps during growth is controlled by carbon source and the composition of growth-depleted medium: DiS- $C_{3}(3)$ fluorescence assay. Int J Biochem Cell Biol. 2005;37(12):2536-43.

43. Chappell J, Vonlanken C, Vogeli U. Elicitor-inducible 3-hydroxy-3-methylglutaryl coenzyme a reductase activity is required for sesquiterpene accumulation in tobacco cell suspension cultures. Plant Physiol. 1991:97(2):693-8.

44. Chappell J, Wolf F, Proulx J, Cuellar R, Saunders C. Is the reaction catalyzed by 3-hydroxy-3-methylglutaryl coenzyme a reductase a rate-limiting step for isoprenoid biosynthesis in plants? Plant Physiol. 1995;109(4):1337-43.

45. Hampton R, Dimster-Denk D, Rine J. The biology of HMG-CoA reductase: the pros of contra-regulation. Trends Biochem Sci. 1996;21(4):140-5.

46. Chappell J, Nable R. Induction of sesquiterpenoid biosynthesis in tobacco cell suspension cultures by fungal elicitor 1. Plant Physiol. 1987;85(2):469-73.

47. Stermer BA, Bostock RM. Involvement of 3-hydroxy-3-methylglutaryl coenzyme a reductase in the regulation of sesquiterpenoid phytoalexin synthesis in potato. Plant Physiol. 1987;84(2):404-8.

48. Chen XF, Huang $C$, Yang $X Y$, Xiong L, Chen XD, Ma LL. Evaluating the effect of medium composition and fermentation condition on the microbial oil production by Trichosporon cutaneum on corncob acid hydrolysate. Bioresour Technol. 2013;143:18-24.

49. Singla A, Verma D, Lal B, Sarma PM. Enrichment and optimization of anaerobic bacterial mixed culture for conversion of syngas to ethanol. Bioresour Technol. 2014;172:41-9.

50. Kapic A, Jones ST, Heindel TJ. Carbon monoxide mass transfer in a syngas mixture. Ind Eng Chem Res. 2006;45(26):9150-5.

51. Akpinar O, Ucar FB. Molecular characterization of Yarrowia lipolytica strains isolated from different environments and lipase profiling. Turk J Biol. 2013;37(3):249-58.

52. Huang C, Wu H, Liu L-P, Lou W-Y, Zong M-H. Effects of alcohol compounds on the growth and lipid accumulation of oleaginous yeast Trichosporon fermentans. PLoS ONE. 2012;7(10):1-12.

53. Huang $C$, Wu H, Liu Q-P, Li Y-Y, Zong M-H. Effects of aldehydes on the growth and lipid accumulation of oleaginous yeast Trichosporon fermentans. J Agric Food Chem. 2011;59(9):4606-13.

54. Huang C, Wu H, Liu ZJ, Cai J, Lou WY, Zong MH. Effect of organic acids on the growth and lipid accumulation of oleaginous yeast Trichosporon fermentans. Biotechnol Biofuels. 2012;5:4.

55. Huang $\mathrm{C}$, Zong $\mathrm{MH}, \mathrm{Wu} \mathrm{H}$, Liu QP. Microbial oil production from rice straw hydrolysate by Trichosporon fermentans. Bioresour Technol. 2009;100(19):4535-8.

56. Zhao X, Kong X, Hua Y, Feng B, Zhao Z. Medium optimization for lipid production through co-fermentation of glucose and xylose by the oleaginous yeast Lipomyces starkeyi. Eur J Lipid Sci Technol. 2008;1 10(5):405-12.

57. Zhu LY, Zong MH, Wu H. Efficient lipid production with Trichosporon fermentans and its use for biodiesel preparation. Bioresour Technol. 2008;99(16):7881-5

58. Jernejc K, Legisa M. The influence of metal ions on malic enzyme activity and lipid synthesis in Aspergillus niger. FEMS Microbiol Lett. 2002;217(2):185-90.

59. Maruyama T, Ito M, Kiuchi F, Honda G. Molecular cloning, functional expression and characterization of D-limonene synthase from Schizonepeta tenuifolia. Biol Pharm Bull. 2001;24(4):373-7.

60. Yuba A, Yazaki K, Tabata M, Honda G, Croteau R. cDNA cloning, characterization, and functional expression of 4S-(-)-limonene synthase from Perilla frutescens. Arch Biochem Biophys. 1996;332(2):280-7.

61. Krause M, Neubauer A, Neubauer P. The fed-batch principle for the molecular biology lab: controlled nutrient diets in ready-made media improve production of recombinant proteins in Escherichia coli. Microb Cell Fact. 2016;15:110.

62. Lisboa P, Rodrigues AR, Martin JL, Simoes P, Barreiros S, Paiva A. Economic analysis of a plant for biodiesel production from waste cooking oil via 
enzymatic transesterification using supercritical carbon dioxide. J Supercrit Fluids. 2014;85:31-40.

63. Zhang $\mathrm{H}$, Wang $\mathrm{Q}$, Mortimer SR. Waste cooking oil as an energy resource: review of Chinese policies. Renew Sustain Energy Rev. 2012;16(7):5225-31.

64. Buczek B. Waste cooking oil as a substrate for biodiesel production. J Agric Sci Technol B. 2014;4(6):493-500.

65. Kulkarni MG, Dalai AK. Waste cooking oil-an economical source for biodiesel: a review. Ind Eng Chem Res. 2006;45(9):2901-13.

66. Bednarski W, Adamczak M, Tomasik J, Plaszczyk M. Application of oil refinery waste in the biosynthesis of glycolipids by yeast. Bioresour Technol. 2004;95(1):15-8.

67. Demirbas MF, Balat M, Balat H. Biowastes-to-biofuels. Energy Convers Manag. 2011;52(4):1815-28.

68. Katre G, Raskar S, Zinjarde S, Kumar VR, Kulkarni BD, RaviKumar A. Optimization of the in situ transesterification step for biodiesel production using biomass of Yarrowia lipolytica NCIM 3589 grown on waste cooking oil. Energy. 2018;142:944-52.

69. Liu X, LV J, Xu J, Zhang T, Deng Y, He J. Citric acid production in Yarrowia lipolytica SWJ-1b yeast when grown on waste cooking oil. Appl Biochem Biotechnol. 2015;175(5):2347-56.

70. Papanikolaou S, Aggelis G. Selective uptake of fatty acids by the yeast Yarrowia lipolytica. Eur J Lipid Sci Technol. 2010;105(11):651-5.

71. Foo JL, Susanto AV, Keasling JD, Leong SS, Chang MW. Whole-cell biocatalytic and de novo production of alkanes from free fatty acids in Saccharomyces cerevisiae. Biotechnol Bioeng. 2017;114(1):232-7.

72. Cao X, Lv YB, Chen J, Imanaka T, Wei $\sqcup$, Hua Q. Metabolic engineering of oleaginous yeast Yarrowia lipolytica for limonene overproduction. Biotechnol Biofuels. 2016;9(1):214.
73. Kolek J, Branska B, Drahokoupil M, Patakova P, Melzoch K. Evaluation of viability, metabolic activity and spore quantity in clostridial cultures during ABE fermentation. FEMS Microbiol Lett. 2016;363(6):fnw031.

74. Yu AQ, Pratomo N, Ng TK, Ling H, Cho HS, Leong SSJ, et al. Genetic engineering of an unconventional yeast for renewable riofuel and biochemical production. J Vis Exp. 2016;115:e54371.

75. Isaac IC, Wootton SA, Johnson TJ, Baldwin EL, Gu L, Karki B, et al. Evaluating the efficacy of genetically engineered Escherichia coli W (ATCC 9637) to produce limonene from industrial sugar beets (Beta vulgaris L.). Ind Crops Prod. 2017;108:248-56.

76. Halfmann C, Gu L, Zhou R. Engineering cyanobacteria for the production of a cyclic hydrocarbon fuel from $\mathrm{CO}_{2}$ and $\mathrm{H}_{2} \mathrm{O}$. Green Chem. 2014;16(6):3175-85.

77. Kiyota H, Okuda Y, Ito M, Hirai MY, Ikeuchi M. Engineering of cyanobacteria for the photosynthetic production of limonene from $\mathrm{CO}_{2}$.J Biotechnol. 2014;185:1-7.

78. Madzak C, Treton B, Blanchin-Roland S. Strong hybrid promoters and integrative expression/secretion vectors for quasi-constitutive expression of heterologous proteins in the yeast Yarrowia lipolytica. J Mol Microbiol Biotechnol. 2000;2(2):207-16.

\section{Publisher's Note}

Springer Nature remains neutral with regard to jurisdictional claims in published maps and institutional affiliations.
Ready to submit your research? Choose BMC and benefit from:

- fast, convenient online submission

- thorough peer review by experienced researchers in your field

- rapid publication on acceptance

- support for research data, including large and complex data types

- gold Open Access which fosters wider collaboration and increased citations

- maximum visibility for your research: over $100 \mathrm{M}$ website views per year

At BMC, research is always in progress.

Learn more biomedcentral.com/submissions 